\title{
Claudin-4 overexpression is associated with epigenetic derepression in gastric carcinoma
}

\author{
Mi Jeong Kwon ${ }^{1, *}$, Seok-Hyung Kim ${ }^{2, *}$, Hae Min Jeong ${ }^{1}$, Hun Soon Jung ${ }^{1}$, Sung-Su Kim ${ }^{1}$, Jae Eun Lee ${ }^{3}$, \\ Myung Chan Gye ${ }^{3}$, Özgür Cem Erkin ${ }^{1}$, Sang Seok Koh ${ }^{4}$, Yoon-La Choi ${ }^{2}$, Cheol Keun Park ${ }^{2}$ and Young Kee Shin ${ }^{1,5}$
}

The tight junction (TJ) protein claudin-4 is aberrantly upregulated in gastric cancer, but its clinical significance and the molecular mechanisms underlying claudin-4 overexpression in gastric cancer remain unclear. Here, we investigated its roles and epigenetic mechanisms regulating CLDN4 expression in gastric cancer. We show that increased membranous expression of claudin-4 in gastric carcinoma is associated with better patient prognosis, whereas cytoplasmic claudin-4 expression did not show a significant association with prognosis. Consistent with the correlation of increased membranous claudin- 4 with favorable clinicopathological factors, claudin- 4 overexpression inhibited the migration and invasion of gastric cancer cells; in contrast, it did not affect cell growth. Claudin-4 expression also increased the barrier function of TJs. Claudin-4 upregulation was strongly correlated with DNA hypomethylation in both gastric tissues and gastric cancer cells. Moreover, CLDN4 expression was repressed in normal gastric tissues in association with bivalent histone modifications, and loss of repressive histone methylations and gain of active histone modifications were associated with CLDN4 overexpression in gastric cancer cells. Interestingly, CLDN4 repression could be markedly derepressed by combined treatments that simultaneously target both histone modifications and DNA demethylation in CLDN4-hypermethylated cells, whereas concomitant changes in histone methylations and acetylations are required for CLDN4 induction in CLDN4-repressed cells with low DNA methylation. Taken together, this study reveals that membranous claudin-4 expression is associated with gastric cancer progression and that it is an independent positive prognosis marker in gastric carcinoma. Furthermore, our findings suggest that epigenetic derepression may be a possible mechanism underlying CLDN4 overexpression in gastric cancer and that claudin-4 may have potential as a promising target for the treatment of gastric cancer.

Laboratory Investigation (2011) 91, 1652-1667; doi:10.1038/labinvest.2011.117; published online 15 August 2011

KEYWORDS: claudin-4; DNA methylation; epigenetic derepression; gastric carcinoma; histone modification

Loss of cell polarity with disruption of cell-cell junctions is frequently found in tumor cells and have a crucial role in cancer progression. Tight junctions (TJs), which are one of the structures within the apical junctional complex, act as barriers in epithelial and endothelial cells by mediating adhesion between cells, and tumor cells often exhibit a loss of functional TJs. ${ }^{1,2}$ Moreover, TJs are involved in other functions including cell proliferation and differentiation by recruiting signaling molecules. ${ }^{2}$

Claudins comprising a 24 -member family, ${ }^{3}$ are major integral membrane TJ proteins, and exhibit tissue-specific expression pattern. $^{1}$ Different claudin subtypes are coexpressed in specific cell types and it is general that multiple claudin subtypes contribute to the formation of TJs. ${ }^{1}$ However, the expression of claudins is altered, both elevated and downregulated in several cancers compared with their normal counterparts. ${ }^{1,4,5}$ Claudin-1, claudin-3, claudin-4 and claudin-7 are among the most frequently deregulated claudins in several malignancies. In addition, altered expression and cellular localization of claudins were shown to contribute to tumor progression by affecting cell proliferation, ${ }^{6}$ cell invasion activities ${ }^{7-9}$ and metastatic behavior. ${ }^{10}$

\footnotetext{
${ }^{1}$ Department of Pharmacy, College of Pharmacy, Seoul National University, Seoul, Korea; ${ }^{2}$ Department of Pathology, Samsung Medical Center, Sungkyunkwan University School of Medicine, Seoul, Korea; ${ }^{3}$ Department of Life Science, Hanyang University, Seoul, Korea; ${ }^{4}$ Therapeutic Antibody Research Center, Korea Research Institute of Bioscience and Biotechnology, Daejeon, Korea and ${ }^{5}$ Advanced Institutes of Convergence Technology, Suwon, Kyeonggi-do, Korea

Correspondence: Professor YK Shin, MD, PhD, Laboratory of Molecular Pathology and Cancer Genomics, Department of Pharmacy, College of Pharmacy, Seoul National University, 599 Gwanak-ro, Gwanak-gu, Seoul, 151-742, Republic of Korea.

E-mail:ykeeshin@snu.ac.kr

*These authors contributed equally to this work.

Received 24 September 2010; revised 18 April 2011; accepted 12 May 2011
} 
In particular, claudin- 3 and claudin- 4 have been found highly overexpressed in a wide variety of cancers including breast, ovarian, pancreas and prostate. ${ }^{1-15}$ In stomach cancer, claudin- 4 was identified as one of the markers of gastric adenocarcinoma precursor lesions ${ }^{16}$ and found to be most prominently overexpressed among claudins in gastric adenocarcinoma. ${ }^{17}$ Moreover, its expression was reported to be lower in poorly differentiated diffuse-type gastric cancer than in well-differentiated intestinal-type gastric cancer, suggesting its possible role in determining the diffuse phenotype. ${ }^{17-19}$ In addition to its aberrant expression in several cancers, claudin- 4 expression has been shown to be associated with prognosis in breast or bladder cancer, ${ }^{20,21}$ and has a role in invasiveness and metastatic potential of pancreatic cancer cell, ${ }^{22}$ as well as it induces angiogenic factors in ovarian cancer. ${ }^{23}$ However, although there have been a few studies into the association of claudin- 4 expression and prognosis, it remains unclear whether claudin-4 expression is associated with clinical outcomes in gastric cancer. Although moderateto-strong claudin-4 staining was shown to be associated with decreased survival in the study by Resnick et $a l^{24}$ a recent study by Ohtani et al, ${ }^{25}$ reported that low claudin-4 expression was independently associated with a significant decrease in overall survival. On the other hand, another study showed no significant correlation between claudin-4 expression and patient survival. ${ }^{17}$ Furthermore, the role of claudin-4 expression in gastric cancer is even less well understood.

It is becoming clear that epigenetic changes, in addition to classical genetic abnormalities, are involved in tumorigenesis through their regulation of expression of tumor-suppressor genes or oncogenes. ${ }^{26,27}$ Two epigenetic mechanisms, DNA methylation and histone modifications, are now well recognized as predominant contributors to the silencing of tumor-suppressor genes. ${ }^{28}$ Epigenetic alterations in multiple genes are known to have a role in stepwise gastric tumorigenesis, ${ }^{29,30}$ in addition to the reported involvement of genetic alterations in TP53, E-cadherin, TGF- $\beta$ receptor II and $A P C / \beta$-catenin. ${ }^{31}$ Inactivation of several genes by DNA hypermethylation (for example, APC, E-cadherin, p14, p15, p16) has been reported in gastric cancer. ${ }^{30}$ Similarly, alteration of claudin-4 expression in bladder carcinoma was found to be associated with DNA methylation, ${ }^{21}$ and epigenetic modifications including histone acetylation and DNA demethylation have been reported to be involved in the increase of CLDN4 expression in ovarian cancer cells. ${ }^{32,33}$ Interestingly, our recent study ${ }^{33}$ revealed that claudin-4 overexpression during ovarian tumorigenesis is associated with loss of repressive histone methylations, suggesting that epigenetic derepression also contributes to tumorigenesis by activation of cancer-promoting genes. However, the molecular mechanism responsible for the alteration of claudin- 4 expression during gastric tumorigenesis is unclear. Therefore, we investigated the underlying mechanism of claudin-4 upregulation and the clinical significance of claudin- 4 overexpression in gastric carcinoma.
In this study, we found that increased claudin- 4 expression suppresses cell invasion and migration, suggesting its role in gastric cancer progression, and epigenetic derepression is one possible mechanism underlying claudin- 4 upregulation in gastric cancer.

\section{MATERIALS AND METHODS Patient Information, Human Tissues and Immunohistochemistry}

A total of 485 gastric tissue samples were obtained from patients with gastric carcinoma and other gastric lesions after ethical approval by the institutional review board of the Samsung Medical Center (Seoul, Korea) (for details, see Supplementary Information). Tissue microarray (TMA) slides were prepared for histopathological analysis as described previously. ${ }^{34}$ For immunohistochemistry, TMA sections were incubated with monoclonal claudin- 4 antibody (1:40 dilution; Zymed Laboratories, San Francisco, CA; 18-7341). In this study, the scoring method described by Sinicrope $e t a l^{35}$ was used to evaluate the intensity of the immunohistochemical staining and the proportion of stained epithelial cells was determined as described previously. ${ }^{34}$

\section{Cell Lines and Drug Treatment}

In all, 14 gastric cancer cell lines, AGS, KATOIII, MKN1, MKN28, MKN74, NCI-N87, SNU5, SNU216, SNU484, SNU601, SNU620, SNU638, SNU668 and SNU719 (purchased from the Korean Cell Line Bank, Seoul, Korea) were maintained under appropriate medium conditions. Cells were seeded 1 day before drug treatment. Seeded cells were treated with $5 \mu \mathrm{M}$ 3-deazaneplanocin A (DZNep; obtained from Dr Victor E Marquez, National Cancer Institute, Frederick, MD, USA), 5 or $1 \mu \mathrm{M} 5$-aza-2'-deoxycytidine (5-aza-dC; Sigma, St Louis, MO) or 200-300 nM trichostatin A (TSA; Sigma) as described previously. ${ }^{33}$

\section{Generation of Cell Lines Stably Overexpressing Claudin-4}

Gastric cancer cells (SNU638 and MKN1) were transfected with pCMV-Tag2B vector (empty vector) or claudin-4 expressing pCMV-Tag2B-CLDN4 plasmids using Lipofectamine LTX (Invitrogen, Carlsbad, CA) following the manufacturer's protocol, and transfected cells were selected with G418 (Invitrogen). Claudin-4-overexpressing stable clones were screened and identified by both flow cytometry and western blot. The claudin-4-expressing construct was prepared as described in Supplementary Information.

\section{Flow Cytometry}

Cells were harvested and counted to obtain a cell concentration of $1 \times 10^{6}$ cells per ml PBS buffer supplemented with $0.5 \%$ BSA and $0.1 \%$ sodium azide. After centrifugation, cells were resuspended in $90 \mu \mathrm{l}$ PBS buffer (with $0.5 \%$ BSA and $0.1 \%$ sodium azide) and incubated on ice for $10 \mathrm{~min}$ with $10 \mu \mathrm{l}$ of FcR blocking reagent (Miltenyi Biotec, Auburn, 
CA). APC-conjugated mouse monoclonal anti-human claudin-4 antibody (R\&D Systems, Minneapolis, MN; FAB4219A) or mouse IgG2A isotype control (R\&D Systems) were then added in Fc-blocked cells $\left(1 \times 10^{5}\right)$ and incubated on ice for $45 \mathrm{~min}$ in the dark. After washing cells three times with PBS buffer, cells were also stained with both Annexin V-FITC and propidium iodide (PI) (BD Biosciences, San Diego, CA) for cell viability and finally analyzed on $\mathrm{BD}$ FACSCalibur flow cytometer (BD Biosciences). The percentage of viable cells bearing cell surface claudin- 4 was quantified.

\section{Cell Migration and Invasion Assay}

The migration of empty vector-transfected and claudin-4overexpressing clones was determined using Costar transwell chambers with $8-\mu \mathrm{m}$ diameter pores (Corning, NY) as described in Supplementary Information. For the invasion assay, invaded cells through the ECMatrix-coated membrane were quantified by fluorescence using QCM ECMatrix Cell Invasion Assay kit (Millipore, Temecula, CA; ECM554) following the manufacturer's instructions.

\section{Gelatin Zymography}

Empty vector-transfected or claudin-4-overexpressing cells were serum starved for $24 \mathrm{~h}$. The conditioned medium was concentrated using Amicon Ultra-15 (Millipore, Carrigtwahill, Cork, Ireland) and the protein in the concentrated medium was used for subsequent gelatin zymography as described in Supplementary Information. The gels were then stained with Comassie Blue and destained until clear bands were seen. Gelatinolytic activity was detected as clear bands on a blue background in a stained gel.

\section{TER Measurement}

Empty vector-transfected or claudin-4-overexpressing clones of SNU638 cells were seeded at a density of $1 \times 10^{5}$ on collagencoated Costar transwell membranes $(0.4-\mu \mathrm{m}$ pore size, $12-\mathrm{mm}$ diameter, Corning). Transepithelial resistance (TER) was measured using a Millicell-ERS V-Ohmmeter (Millipore). The obtained values are presented as resistance in Ohms multiplied by the area of the filter after subtraction of blank values.

\section{Quantitative RT-PCR and Western Blotting}

Total RNA was isolated and cDNA synthesized as described previously. ${ }^{33}$ Transcript levels of each gene were determined by quantitative real-time reverse transcription-PCR (RTPCR) as described previously ${ }^{33}$ using the PCR primers and probes listed in Supplementary Table S1.

Whole-cell lysates were extracted using RIPA buffer and they $(50 \mu \mathrm{g})$ were used for immunoblotting using primary antibodies against claudin-4, EZH2, trimethylated histone $\mathrm{H} 3$ lysine 27 (H3K27me3) and $\beta$-actin described in our previous study ${ }^{33}$ according to the standard procedures.

\section{Methylation-Specific PCR and Bisulfite Sequencing PCR} Genomic DNA was extracted from gastric cancer cells, normal gastric frozen tissues or gastric formalin-fixed, paraffin-embedded (FFPE) tissues, and bisulfite modified as described previously. ${ }^{33}$ For genomic DNA preparation from gastric FFPE tissues, the fields of interest were microdissected using a 27-G needle under a dissection microscope. Bisulfite-modified DNA was amplified by methylationspecific PCR (MSP), quantitative MSP (qMSP) and bisulfite sequencing PCR (BSP) using the primers and probes shown in Supplementary Table S1.

\section{ChIP Assay}

Chromatin immunoprecipitation (ChIP) assays were performed using EZ-ChIP kit (Millipore) for cell lines, and EpiQuik Tissue Chromatin Immunoprecipitation Kit (Epigentek, Brooklyn, NY) for normal gastric frozen tissues according to the manufacturer's protocol. Normal gastric tissues from gastric cancer patients which were confirmed not to include gastric lesions such as carcinoma by pathological evaluation were used in ChIP experiments. For ChIPs using frozen tissues, $10 \mathrm{mg}$ tissues per one ChIP were used. The antibodies against various histone marks as described previously ${ }^{33}$ were used for ChIP analyses, and quantification of ChIP results was performed by qPCR. The sequences of the probe and primers for ChIP and quantitative ChIP (qChIP) are provided in Supplementary Table S1.

\section{siRNA Transfection}

The small-interfering RNA (siRNA) targeting EZH2 and non-targeting control were purchased from Dharmacon (Lafayette, CO) and cells were treated with $100 \mathrm{nM}$ final concentration of siRNA using Oligofectamine (Invitrogen) following the manufacturer's instructions.

Figure 1 Expression of claudin-4 in gastric tissues and its association with gastric carcinoma patients' survival. (a) Expression profile of claudin-4 in the normal gastric epithelium, gastric adenocarcinoma and its related lesions including high/low-grade gastric epithelial dysplasia, intestinal metaplasia and chronic gastritis. Images of representative immunohistochemical staining with anti-claudin-4 of gastric tissues are shown (upper). Claudin-4 expression in cell membranes and cytoplasm was scored based on immunohistochemical staining levels (lower). A total of 485 samples including the normal gastric epithelium (NL, $n=23$ ), chronic atrophic gastritis (CG, $n=18$ ), intestinal metaplasia (IM, $n=49$ ), low-grade dysplasia (LD, $n=25)$, high-grade dysplasia (HD, $n=25)$ and gastric adenocarcinoma (CA, $n=345)$ were used for immunohistochemistry analysis, and the immunoreactive score (IS, $0 \leq \mathrm{IS} \leq 12)$ for each type of lesion is shown (lower). Comparisons of IS values between the two groups were performed using Student's $t$-test (two-sided). (b) Association of membranous claudin-4 expression with patients' survival in total gastric adenocarcinoma or (c) in intestinal-type gastric adenocarcinoma. Survival durations of gastric adenocarcinoma patients were calculated using Kaplan-Meier survival analyses. Log-rank tests were used to compare the survival curves between three groups: high expression (IS $\geq 9)$, intermediate $(3 \leq I S \leq 8)$ and low $(0 \leq I S \leq 2)$. 


\section{Statistical Analysis}

Statistical analyses were performed using Fisher's exact tests, Pearson's $\chi^{2}$ tests, ANOVA, Student's $t$-test, Mann-Whitney tests and correlation analysis. Kaplan-Meier analysis, logrank test, univariate and multivariate survival analyses using the Cox's regression model were also conducted. All statistical a

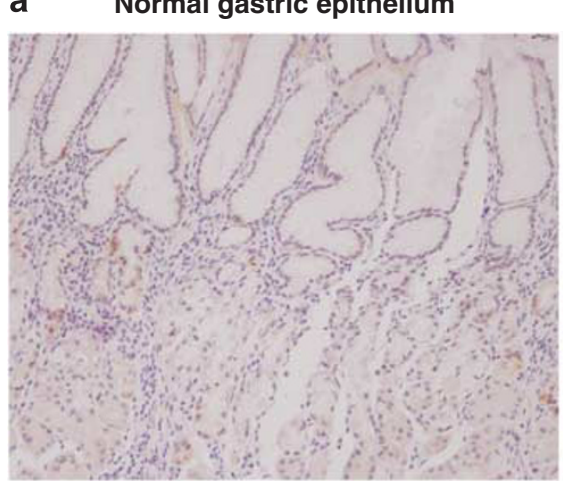

Low grade dysplasia

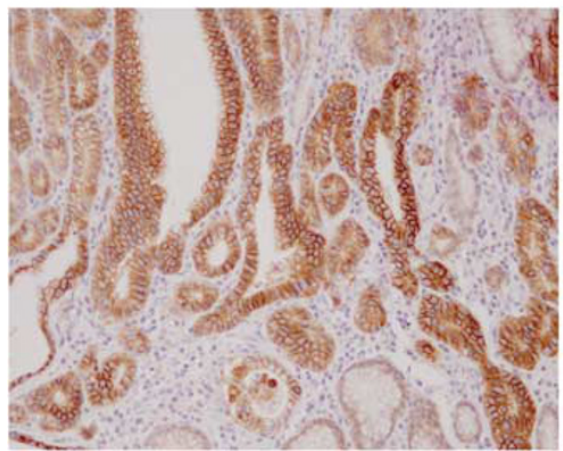

Chronic gastritis

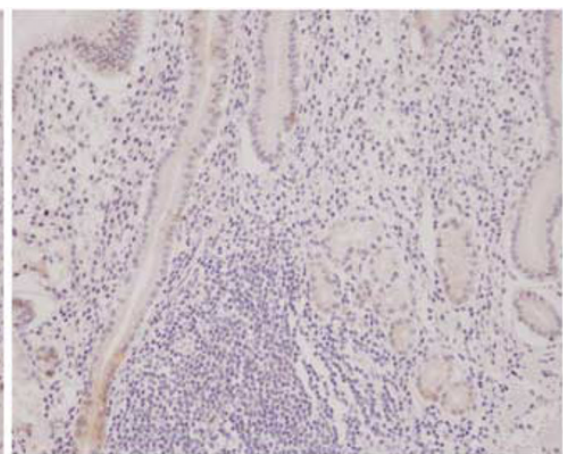

High grade dysplasia

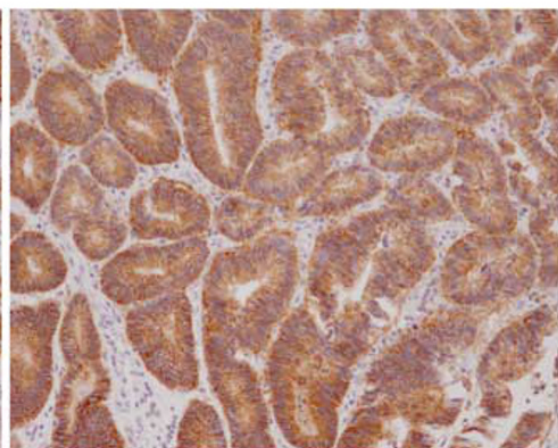

Intestinal metaplasia

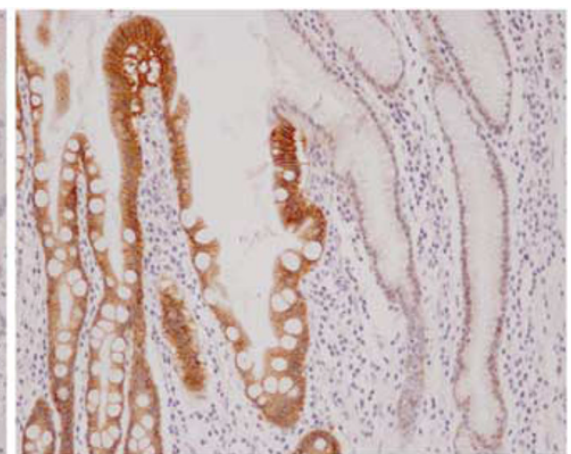

Adenocarcinoma

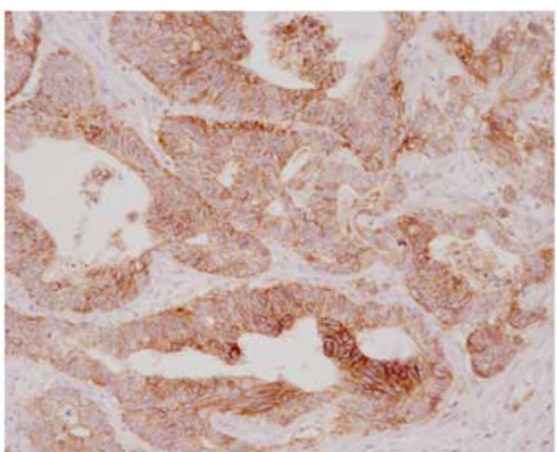

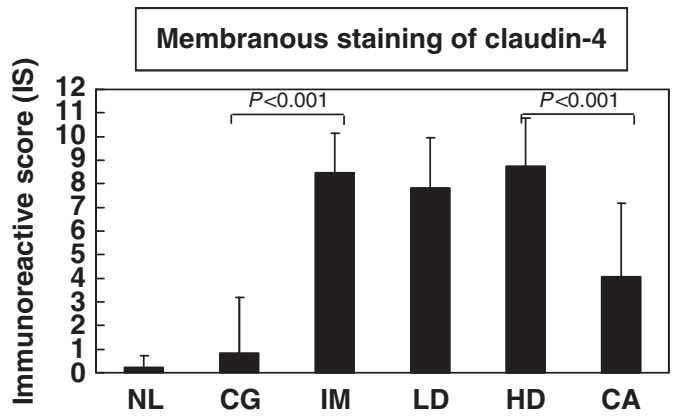

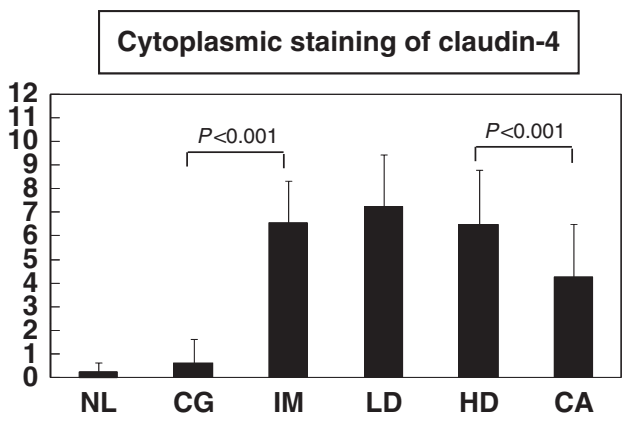

b

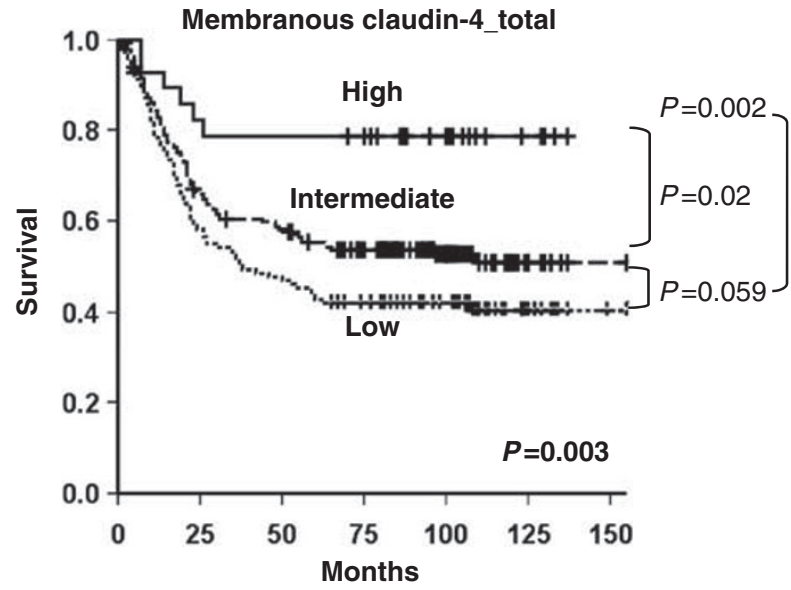

C

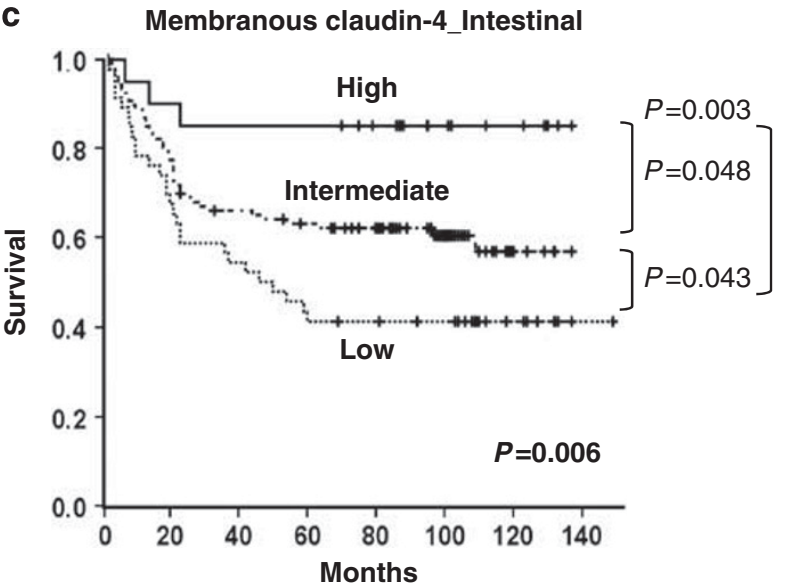


analyses were performed using SPSS 12.0 or 18.0 statistic software for Windows (SPSS, Chicago, IL). $P$-values $\leq 0.05$ were considered statistically significant.

\section{RESULTS}

Increased Membranous Claudin-4 Expression in Gastric Adenocarcinoma Correlates with Favorable Clinicopathological Parameters

The gene expression of several claudins in normal and cancerous stomach tissues was determined using microarray data based on Affymetrix HG-U133, which is used for our previous studies ${ }^{15,36}$ (Supplementary Table S2). In stomach cancer, CLDN4 was the most upregulated claudin gene compared with normal gastric tissue, although the expression of other claudin genes including CLDN1, CLDN3 and CLDN7 was also elevated in gastric carcinoma consistent with previous studies. ${ }^{24,37}$ In contrast, CLDN18 expression was significantly downregulated in gastric carcinoma as reported previously. ${ }^{38}$

On the basis of these microarray data, the protein levels of claudin-4 expression were further determined in normal gastric mucosa, gastric adenocarcinoma and its related lesions using immunohistochemistry. Claudin-4 was strongly stained in both cytoplasm and membrane in intestinal metaplasia and gastric epithelial dysplasia lesions, whereas normal gastric epithelia and chronic gastritis samples were focally and weakly stained for claudin-4 (Figure 1a, Supplementary Table S3). These results are consistent with those in previous studies, ${ }^{16,39}$ and further support the suggestion of usefulness of claudin- 4 expression as a marker of gastric adenocarcinoma precursor lesions. The majority of gastric adenocarcinoma samples $(336 / 345,97.39 \%)$ exhibited some degree of claudin-4 staining in both membrane and cytoplasm. Overall, both membranous and cytoplasmic staining levels in adenocarcinoma were significantly lower than those in intestinal metaplasia and dysplasia but were higher than those in normal and inflammatory gastric epithelium samples $(P<0.001$, Supplementary Table S3).

Next, the correlations between claudin- 4 expression in gastric carcinoma and various clinicopathological parameters were analyzed. Table 1 shows that increased expression of membranous claudin- 4 is significantly associated with all of the favorable parameters including absence of nodal metastasis $(P=0.001)$, small tumor size $(P=0.02)$, early gastric carcinoma $(P=0.001)$, intestinal-type of Lauren's classification $(P<0.001)$, well or moderate differentiation (low grade) $(P<0.001)$, low depth of tumor invasion $(P=0.008)$ and early clinical staging $(P=0.001)$. In contrast, cytoplasmic claudin-4 expression was not correlated with tumor size, stage, nodal metastasis, early gastric carcinoma and depth of tumor invasion; however, it was associated with well differentiation $(P<0.001)$ and with the intestinal-type of Lauren's classification $(P=0.03)$.
Table 1 Correlation of membranous and cytoplasmic claudin-4 expression levels with clinicopathological features in gastric carcinomas

\begin{tabular}{|c|c|c|c|c|c|}
\hline \multirow[t]{2}{*}{ Variable } & \multirow[t]{2}{*}{ No. } & \multicolumn{4}{|c|}{ Mean of IS } \\
\hline & & $\begin{array}{l}\text { Membrane } \\
\text { staining }\end{array}$ & $P$-value & $\begin{array}{l}\text { Cytoplasmic } \\
\text { staining }\end{array}$ & $P$-value \\
\hline \multicolumn{6}{|l|}{ Age (years) } \\
\hline$<60$ & 166 & $3.49 \pm 2.92$ & \multirow{2}{*}{0.002} & $4.17 \pm 2.40$ & \multirow{2}{*}{0.45} \\
\hline$\geq 60$ & 174 & $4.56 \pm 3.21$ & & $4.36 \pm 2.14$ & \\
\hline \multicolumn{6}{|l|}{ Tumor size $(\mathrm{cm})$} \\
\hline$<5.0$ & 123 & $4.50 \pm 3.11$ & \multirow{2}{*}{0.02} & $4.52 \pm 2.19$ & \multirow{2}{*}{0.10} \\
\hline$\geq 5.0$ & 215 & $3.70 \pm 3.06$ & & $4.10 \pm 2.30$ & \\
\hline \multicolumn{6}{|l|}{ Node metastasis } \\
\hline Negative & 101 & $4.71 \pm 3.20$ & \multirow{4}{*}{0.001} & $4.47 \pm 2.31$ & \multirow{4}{*}{0.35} \\
\hline Positive (1-6) & 59 & $4.41 \pm 3.16$ & & $4.23 \pm 2.11$ & \\
\hline Positive (7-15) & 84 & $3.06 \pm 2.67$ & & $3.92 \pm 1.99$ & \\
\hline Positive ( $\geq 16$ ) & 52 & $3.58 \pm 3.19$ & & $4.46 \pm 2.59$ & \\
\hline \multicolumn{6}{|l|}{ Lauren } \\
\hline Intestinal & 151 & $5.61 \pm 2.98$ & \multirow{3}{*}{$<0.001$} & $4.58 \pm 2.14$ & \multirow{3}{*}{0.03} \\
\hline Diffuse & 169 & $2.67 \pm 2.61$ & & $3.93 \pm 2.32$ & \\
\hline Mixed & 12 & $4.17 \pm 2.73$ & & $4.83 \pm 2.52$ & \\
\hline \multicolumn{6}{|l|}{ Gross type } \\
\hline EGC & 64 & $5.16 \pm 3.19$ & \multirow{2}{*}{0.001} & $4.75 \pm 2.19$ & \multirow{2}{*}{0.056} \\
\hline AGC & 276 & $3.76 \pm 3.04$ & & $4.15 \pm 2.28$ & \\
\hline \multicolumn{6}{|l|}{ WHO classification } \\
\hline Tubular, well & 45 & $6.69 \pm 2.53$ & \multirow{5}{*}{$<0.001$} & $5.69 \pm 2.32$ & \multirow{5}{*}{$<0.001$} \\
\hline Tubular, moderately & 105 & $5.16 \pm 3.04$ & & $4.18 \pm 1.94$ & \\
\hline Tubular, poorly & 115 & $2.96 \pm 2.86$ & & $4.12 \pm 2.24$ & \\
\hline Signet ring cell type & 33 & $2.03 \pm 2.17$ & & $3.42 \pm 2.45$ & \\
\hline Mucinous type & 8 & $1.75 \pm 1.98$ & & $2.88 \pm 2.53$ & \\
\hline \multicolumn{6}{|l|}{ Depth of invasion } \\
\hline 1 & 65 & $5.05 \pm 3.23$ & \multirow{4}{*}{0.008} & $4.62 \pm 2.05$ & \multirow{4}{*}{0.54} \\
\hline$\|$ & 67 & $4.37 \pm 3.09$ & & $4.07 \pm 2.06$ & \\
\hline III & 174 & $3.64 \pm 3.03$ & & $4.23 \pm 2.33$ & \\
\hline IV & 35 & $3.49 \pm 2.87$ & & $4.14 \pm 2.72$ & \\
\hline \multicolumn{6}{|l|}{ pTNM staging } \\
\hline 1 & 87 & $5.18 \pm 3.07$ & \multirow{4}{*}{0.001} & $4.59 \pm 2.16$ & \multirow{4}{*}{0.23} \\
\hline$\|$ & 46 & $3.61 \pm 3.16$ & & $4.26 \pm 2.29$ & \\
\hline III & 115 & $3.78 \pm 2.98$ & & $3.97 \pm 2.08$ & \\
\hline IV & 94 & $3.47 \pm 3.03$ & & $4.33 \pm 2.54$ & \\
\hline
\end{tabular}

AGC, advanced gastric carcinoma; EGC, early gastric carcinoma.

TNM staging was performed according to the staging system of the American Joint Committee on Cancer (AJCC) Sixth edition, which divides them into four categories (stages I-IV). The association of the expression with clinicopathological factors was assessed by Fisher's exact test and Pearson's $\chi^{2}$ test. 


\section{Enhanced Membranous Expression of Claudin-4 Is Associated with Better Prognosis and Longer Survival Time}

The correlation of membranous and cytoplasmic claudin- 4 expressions with overall survival rate of gastric carcinoma patients was also assessed. Membranous claudin-4 expression was significantly associated with patients' survival $(P=0.003$, Figure $1 \mathrm{~b}$ ). The mean survival time for patients with high membranous claudin-4 expression was 111 months, which was significantly higher than the times for both intermediate (91 months) and low (76 months) expression groups (high $v$ intermediate; $P=0.02$, high $v s$ low; $P=0.002$ ). In a separate analysis of the intestinal-type gastric carcinoma samples, higher membranous claudin-4 expression was also significantly associated with longer patients' survival $(P=0.006$, Figure 1c). In contrast, cytoplasmic claudin-4 expression was not significantly associated with patient's survival in intestinal types of gastric carcinoma $(P=0.31)$; however, it did tend to correlate with patients' survival in total gastric carcinoma $(P=0.08$, Supplementary Figure $S 1)$.

Both univariate and multivariate analyses of the overall survival of gastric carcinoma patients was performed using Cox's proportional hazards regression. High expression of membranous claudin-4 was significantly associated with better prognosis $(\mathrm{RR}=0.339, P=0.009)$ in univariate analysis (Table 2). Moreover, it remained as a significant independent prognostic factor $(\mathrm{RR}=0.366, P=0.02)$ after multivariate analysis that included age, histological grade, clinical stage and Lauren's classification (Table 2). In contrast to membranous expression, there was no significant correlation between cytoplasmic claudin-4 expression and patients' prognosis in either univariate or multivariate analysis.

\section{Increased Membranous Claudin-4 Expression Suppresses Migration and Invasion of Gastric Cancer Cells, While It Increases the Barrier Function of TJs}

On the basis of the correlation of claudin-4 expression with clinicopathological features in gastric cancer, the possible role of claudin-4 overexpression was investigated in gastric cancer cells. We established claudin-4-overexpressing clones from two CLDN4-repressed gastric cancer cell lines (SNU638 and MKN1). Two stable clones (CLDN4-1 and CLDN4-2) with a high percentage of cell surface claudin-4-expressing cells and high levels of claudin-4 expression (Figure 2a) were selected from each cell line for additional analyses.

To determine whether claudin-4 expression influences cell growth, the numbers of viable cells were counted. There was no significant difference in cell growth between claudin4-overexpressing clones and empty vector-transfected control cells of both SNU638 and MKN1 cells (Figure 2b), demonstrating that the expression of claudin-4 does not affect cell growth.

We also examined the effect of claudin-4 expression on cell migration and invasion activity in gastric cancer cells. In claudin-4-overexpressing clones of both SNU638 and MKN1 cells, cell migration was significantly decreased compared with empty vector-transfected control cells $(P<0.001$, Figure 2c). In addition, claudin-4-overexpressing clones showed significantly reduced cell invasion compared with empty vector-transfected cells $(P<0.01$, Figure $2 \mathrm{~d})$. However, significantly decreased gelatinolytic matrix metalloproteinase (MMP) activities were not observed in claudin-4-overexpressing SNU638 cells (Figure 2e), indicating that a decrease in invasion resulting from claudin-4 overexpression in SNU638 cells is unlikely to occur in association with MMP activities. Taken together, these results suggest that increased claudin-4 expression inhibits the cell migration and invasion by localizing at membrane regions regardless of MMP activities.

The effect of claudin-4 expression on the intercellular barrier function of TJs was also assessed in gastric cancer cells by measuring TER. As shown in Figure $2 \mathrm{f}$, overexpression of claudin-4 led to a significantly higher level of TER compared with that in empty vector-transfected SNU638 cells $(P<0.01)$, indicating an enhancement of tightness of TJs after claudin-4 overexpression. Immunofluorescence results indicated that transfected claudin-4 expression was located at cell-cell contact sites (Supplementary Figure S2). This results show that claudin-4 expression increases the barrier function of TJs in gastric cancer cells.

\section{Claudin-4 Expression in Gastric Cancer Cells Is Inversely Correlated with DNA Methylation}

As increased claudin-4 expression was shown to inhibit cell invasion and migration as well as to increase the barrier function of TJs, the molecular mechanism regulating claudin-4 expression in gastric cancer was investigated. First, we determined claudin-4 expression at the transcript and protein levels in 14 gastric cancer cell lines to confirm the regulation level. CLDN4 expression was well correlated with the level of claudin-4 protein expression, indicating that claudin4 expression is regulated at the transcript level (Figure 3a, middle). Claudin-4 expression by western blot was little or undetectable in four gastric cancer cells (MKN1, SNU484, SNU638 and SNU668) indicating low CLDN4 transcript levels, whereas the other cells showed high claudin-4 expression.

To determine the transcriptional regulation mechanism of CLDN4 expression in gastric cancer cells, DNA methylation status was assessed by MSP. DNA methylation was found in the CLDN4 promoter in CLDN4-repressed cells, whereas cells in which CLDN4 was highly expressed did not exhibit DNA methylation (Figure 3a, bottom). This indicates that DNA hypermethylation is associated with CLDN4 repression in gastric cancer cells. However, DNA methylation level in the CLDN4 promoter varied in cell lines with low levels of CLDN4 expression. CLDN4 expression was repressed in SNU638 and SNU668 cells despite the relatively low level 
Table 2 Univariate and multivariate analyses of prognostic variables related to overall survival of gastric carcinoma patients using Cox's proportional hazards regression

Predictors
Univariate

Ratio of risk (RR)
$(95 \% \mathrm{Cl})$

1.00

$1.054(0.808 \sim 1.376)$

$60+$

Grade

W/D

M/D

$P / D$

Signet ring $C A$

Mucinous CA

pTNM staging

।

॥

III

IV

Lauren classification

Intestinal

Diffuse

Mixed

Claudin-4 (m)

Low $(<9)$

High $(\geq 9)$

Claudin-4 (c)

Low $(<7)$

High $(\geq 7)$
0.70

$<0.001$

1.00

$1.549(0.710 \sim 3.380)$

$1.318(0.466 \sim 3.726)$

$1.782(0.590 \sim 5.381)$

$0.983(0.241 \sim 4.012)$

Multivariate

Ratio of risk (RR)

$(95 \% \mathrm{Cl})$

1.00

1.332 (0.975 1.819)
$4.068(2.199 \sim 7.527)$

$5.227(2.862 \sim 9.545)$

7.907 (4.017 15.564)

$5.574(1.963 \sim 15.830)$

$<0.001$

1.00

$2.195(1.038 \sim 4.641)$

9.277 (5.424 15.867)

$27.054(15.891 \sim 46.057)$

1.00

$1.974(1.479 \sim 2.635)$

$1.100(0.478 \sim 2.531)$

1.00

0.50

$<0.001$

1.00

$1.172(0.454 \sim 3.026)$

5.206 (2.503 10.089)

15.281 (7.591 30.758)

$<0.001$

1.00

$1.238(0.531 \sim 2.887)$

$0.469(0.159 \sim 1.391)$

0.009

1.00

$0.366(0.157 \sim 0.852)$

$0.339(0.150 \sim 0.766)$

0.21

1.00

$0.834(0.518 \sim 1.340)$

c, cytoplasmic staining; $m$, membrane staining; $M / D$, moderately differentiated; mucinous $C A$, mucinous carcinoma; $P / D$, poorly differentiated; signet ring CA, signet ring cell carcinoma; W/D, well differentiated.

Figure 2 Effects of claudin-4 overexpression on cell growth, migration and invasion in gastric cancer cells. (a) Claudin-4 expression in claudin-4-overexpressing clones of SNU638 and MKN1 cells. The percentage of cell surface claudin-4-positive cells among viable cells (Annexin V and PI negative) and claudin-4 expression in stably transfected cells with empty vector (EV) or claudin-4-expressing plasmids (CLDN4-1 and CLDN4-2) were determined using flow cytometry (upper) and western blot (lower). (b) Effect of claudin-4 overexpression on cell growth. An equal number of empty vector control or claudin-4-overexpressing clones were plated and cell numbers were determined by counting the viable cells after trypan blue staining at each time point. Cell numbers represent mean \pm s.d. of two independent experiments in duplicate. (c) Effect of claudin-4 expression on cell migration. (d) Effect of claudin-4 expression on cell invasion activity. Migration and invasion activity are represented as relative percentage compared with control cells and these experiments were repeated three times with duplicate in each experiment. (e) MMP activities (MMP-2 and MMP-9) were compared between empty vector control and claudin-4-overexpressing clones of SNU638 cells. MMP activities were determined using gelatin zymography. Gelatinolytic activity was detected as clear bands on a blue background in a stained gel. (f) Effect of claudin-4 overexpression on TER. TER values were taken for 12,24 and $48 \mathrm{~h}$ after seeding of claudin-4-overexpressing clone or empty vectortransfected SNU638 cells and are represented as mean \pm s.d. values from three independent experiments. ${ }^{\star} P \leq 0.05,{ }^{\star *} P \leq 0.01,{ }^{\star * *} P \leq 0.001$ (Student's $t$-test). 
of CLDN4 methylation, whereas CLDN4 expression was repressed in SNU484 and MKN1 cells with DNA hypermethylation, suggesting that another mechanism is involved in CLDN4 repression of gastric cells with low DNA methylation.

\section{Increased Claudin-4 Expression During Gastric} Tumorigenesis Is Associated with DNA Hypomethylation To further explore the relationship between DNA methylation and alteration of claudin-4 expression during gastric tumorigenesis, the methylation profile was analyzed in gastric a

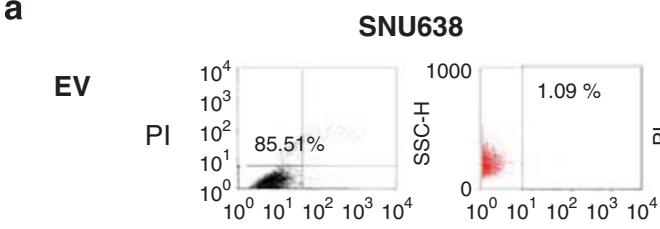

CLDN4-1

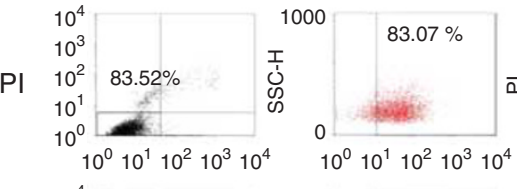

CLDN4-2

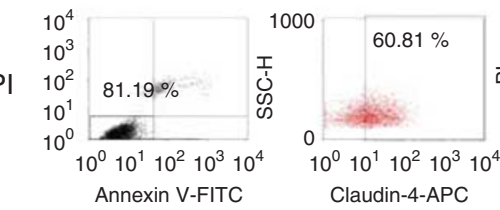

EV CLDN4-1 CLDN4-2

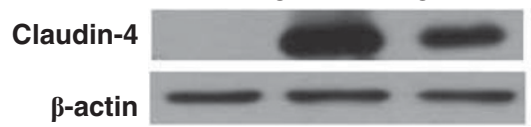

MKN1
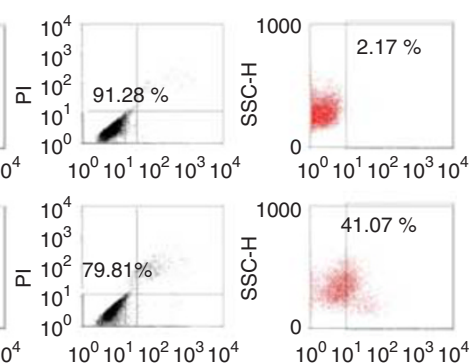

$10^{0} 10^{1} 10^{2} 10^{3} 10^{4}$
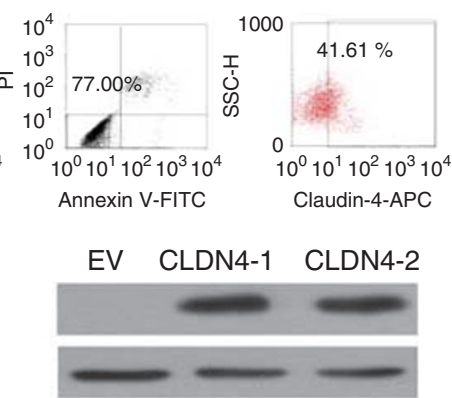
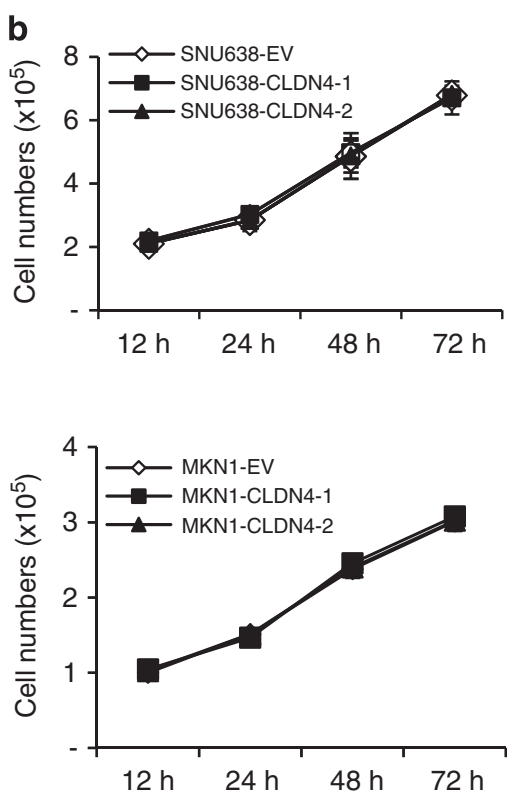

c

SNU638

MKN1

d

SNU638
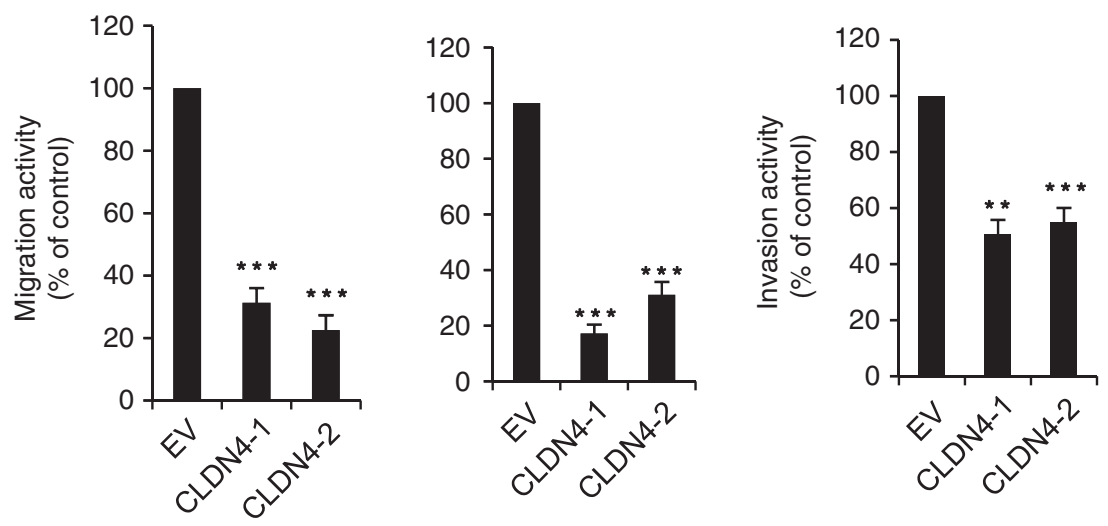

MKN1

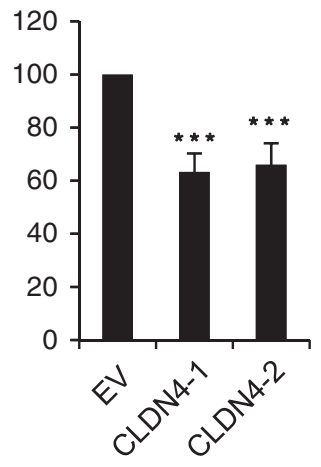

e

SNU638

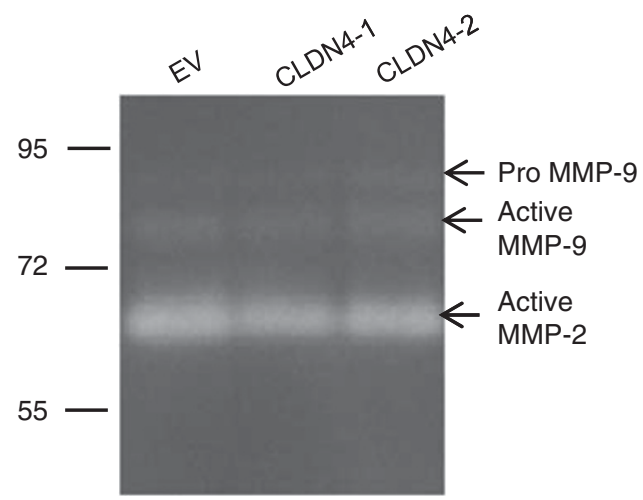

f

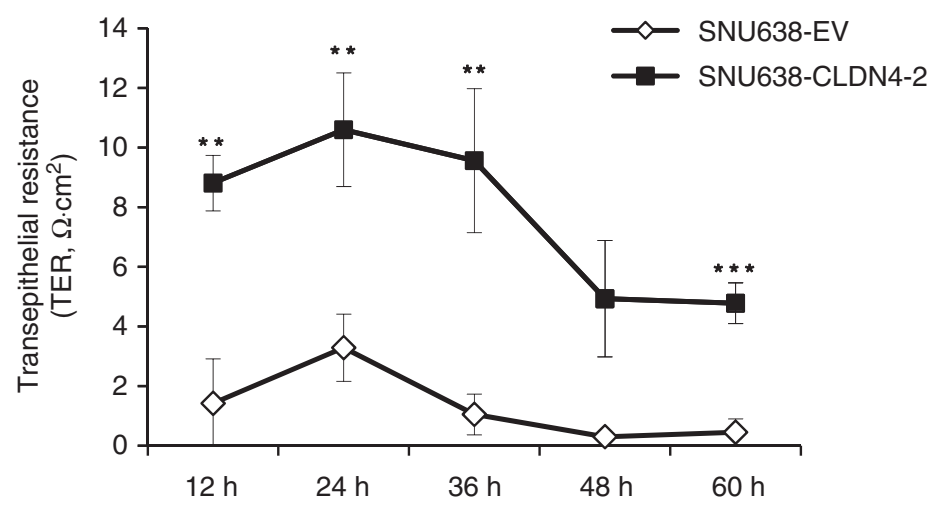


a
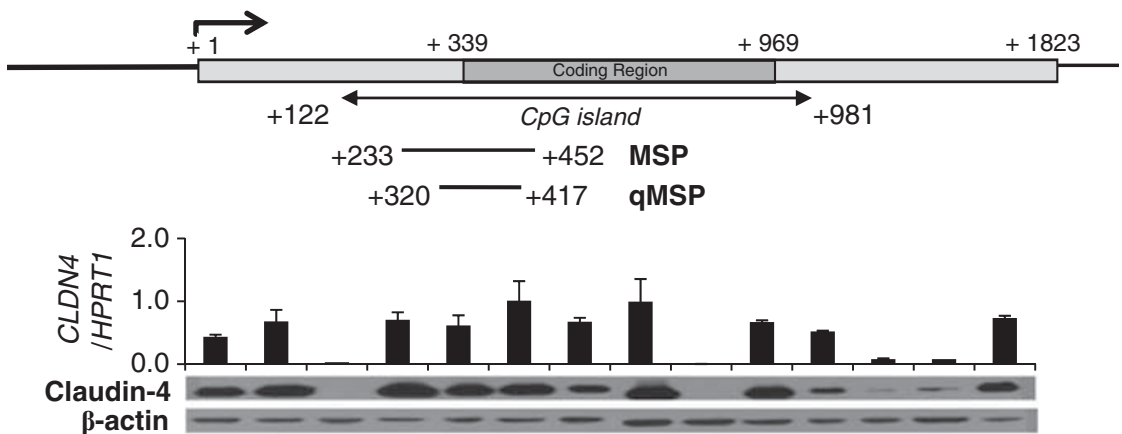

$\beta$-actin

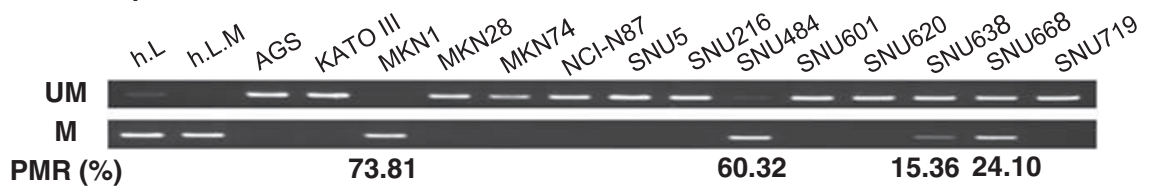

b

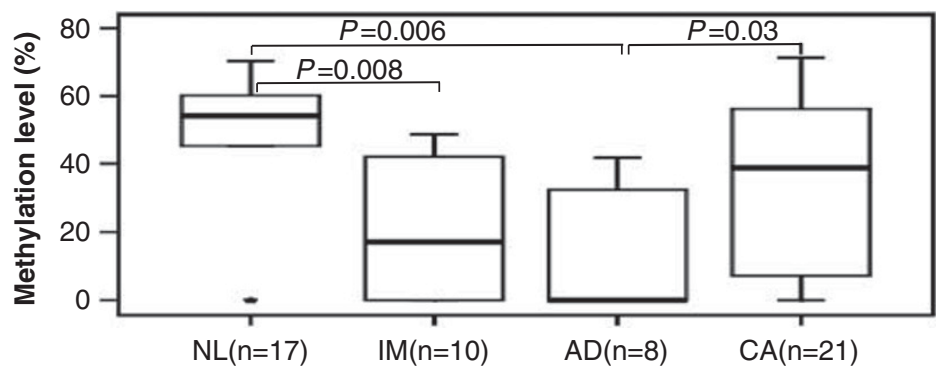

Gastric FFPE tissues

C
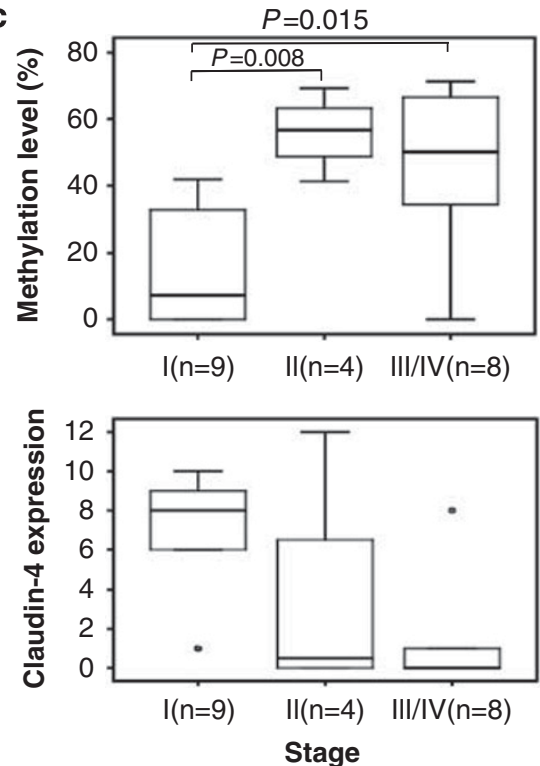

d

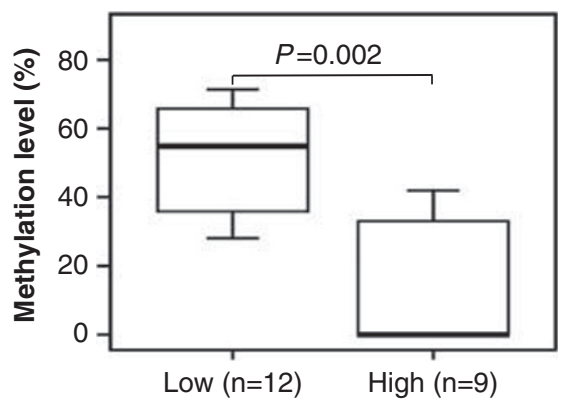

Claudin-4 expression

Figure 3 Status of DNA methylation at the CLDN4 promoter in gastric cancer cells and gastric tissues. (a) Relationship between claudin-4 expression and DNA methylation in gastric cancer cells. Locations of primers used for MSP analyses (top) in the CLDN4 sequence are shown. CpG island in the promoter was predicted, and primers and probes for MSP and qMSP were designed using Methprimer or by TIB MOLBIOL (Berlin, Germany) as described previously. ${ }^{33}$ The transcription start site is indicated by +1 . Claudin-4 expression at the transcript level was determined by qRT-PCR as the relative expression to HPRT1 expression, and at the protein level by immunoblotting (middle). MSP was performed using bisulfite-modified genomic DNA of gastric cancer cell lines for DNA unmethylated (UM) and DNA methylated (M) (bottom). Sss-I treated (assumed as 100\% methylated) human leukocytes were the positive control. PMR (percentage of methylated reference, \%) was determined by qMSP using a Taqman probe and calculated as described previously. ${ }^{33}$ (b) CLDN4 DNA methylation in gastric FFPE tissues. DNA methylation level is represented as the average PMR values of two to five repeated qMSP assays. Box plot depicts the median value as a horizontal line within box, the 25th and 75th percentile value as the bottom and top of box, respectively. AD: gastric adenoma. (c) DNA methylation of gastric carcinoma FFPE tissues by stages. DNA methylation level (upper) and claudin-4 expression (lower) are shown. (d) Comparison of DNA methylation level between the low claudin-4-expressing and the high claudin-4-expressing groups in gastric carcinoma FFPE tissues. Differences in DNA methylation between two groups were analyzed using non-parametric Mann-Whitney test. 
FFPE tissues, including the normal stomach, intestinal metaplasia, gastric adenoma and gastric carcinoma. DNA methylation of the CLDN4 promoter was frequently observed in normal gastric tissues $(76.47 \%, 13 / 17)$ with high levels (median PMR, 54.03\%) (Supplementary Table S4), and CLDN4 DNA methylation levels were significantly lower in intestinal metaplasia $(P=0.008)$ and adenoma $(P=0.006)$ compared with the normal gastric epithelium (Figure $3 \mathrm{~b}$ ), showing that CLDN4 is DNA hypomethylated during early gastric tumorigenesis. In addition, lower CLDN4 DNA methylation in intestinal metaplasia and adenoma was significantly correlated with higher claudin- 4 expression in those tissues $(P<0.05$, Supplementary Table S5), indicating that claudin- 4 overexpression in gastric precancerous lesions is related to promoter DNA demethylation.

Levels of CLDN4 DNA methylation in gastric carcinoma were greater than those in adenoma. Interestingly, CLDN4 DNA methylation in advanced stages of gastric carcinoma was significantly higher than that in early stages of carcinoma $(P<0.05$, Figure $3 c)$, showing that CLDN4 DNA methylation is increased with the progression of gastric carcinoma. We also assessed the association between DNA methylation and claudin-4 expression in gastric carcinoma tissues based on cell line data. Both DNA methylation level and frequency were significantly higher in the claudin- 4 low-expressing group $(P=0.002$ for methylation level; Figure $3 \mathrm{~d}, P=0.006$ for frequency; Supplementary Table S6), and claudin-4 expression showed a significant inverse correlation with DNA methylation (Supplementary Table S5), confirming the presence of a correlation between increased DNA methylation and decreased claudin-4 expression in the progression of gastric carcinoma. Taken together, our data demonstrate that claudin- 4 overexpression during early gastric tumorigenesis is significantly correlated with DNA hypomethylation. Moreover, our results show that decreased claudin-4 expression in the advanced stage of gastric carcinoma compared with the early stage of gastric carcinoma is significantly associated with increased DNA methylation.

\section{Bivalent Histone Modifications Are Associated with CLDN4 Repression in Normal Gastric Tissues}

Although a significant inverse correlation between DNA methylation and claudin-4 expression during gastric tumorigenesis was shown, we found some gastric tissues in which their significant correlation is not observed, for example, claudin-4 expression was not detected in some normal gastric FFPE tissues with a lack of DNA methylation (Supplementary Table S4), consistent with findings in previous gastric cancer cells. Therefore, we further investigated the additional mechanisms involved in the repression of claudin4 expression in normal gastric tissues.

We performed ChIP assays to determine whether histone modifications are related to CLDN4 expression in normal gastric tissues. Normal gastric tissues with relatively low transcript levels of claudin-4 showed different DNA methylation status in the promoter (Figures $4 \mathrm{a}$ and $\mathrm{b}$ ). ChIPs for repressive histone marks (H3K27me3, dimethylated or trimethylated $\mathrm{H} 3$ lysine 9; $\mathrm{H} 3 \mathrm{~K} 9 \mathrm{me} 2$ or $\mathrm{H} 3 \mathrm{~K} 9 \mathrm{me} 3$ and trimethylated histone $\mathrm{H} 4$ lysine 20; H4K20me3), as well as for the active histone marks (trimethylated histone $\mathrm{H} 3$ lysine 4; H3K4me3, acetylated histone $\mathrm{H} 3$ and $\mathrm{H} 4 ; \mathrm{H} 3 \mathrm{Ac}$ and $\mathrm{H} 4 \mathrm{Ac})$ were carried out in two regions of the CLDN4 promoter in three normal gastric frozen tissues. In accordance with our previous study in normal ovarian cells, ${ }^{33}$ bivalent histone modifications, coexistence of active $\mathrm{H} 3 \mathrm{~K} 4 \mathrm{me} 3$ and repressive $\mathrm{H} 3 \mathrm{~K} 27 \mathrm{me} 3$, were found in the promoter region of CLDN4 in all normal gastric tissues (Figure 4c). High levels of repressive H4K20me3 were also detected in all normal tissues. Interestingly, CLDN4 expression was repressed in normal gastric tissues showing low DNA methylation and tissues with high DNA methylation, suggesting that bivalent histone modifications are crucial for CLDN4 repression in normal gastric tissues independent of DNA methylation.

\section{Decreased Repressive Histone Marks (H3K27me3 and H4K2Ome3) and Increased Active Histone Marks (H3K4me3 and H4Ac) Are Associated with CLDN4 Overexpression in Gastric Cancer Cells}

We also investigated the association of histone modifications in the CLDN4 expression in gastric cancer cells. We detected an abundant enrichment of the active $\mathrm{H} 3 \mathrm{~K} 4 \mathrm{me} 3$ and $\mathrm{H} 3 \mathrm{Ac}$ in the CLDN4 promoter of CLDN4-high expressing NCI-N87 cells, whereas repressive $\mathrm{H} 3 \mathrm{~K} 27 \mathrm{me} 3, \mathrm{H} 3 \mathrm{~K} 9 \mathrm{me} 2$ and $\mathrm{H} 3 \mathrm{~K} 9 \mathrm{me} 3$ were minimally detectable in the promoter (Figure $4 \mathrm{~d})$. On the contrary, a higher level of H3K27me3 and $\mathrm{H} 4 \mathrm{~K} 20 \mathrm{me} 3$, and weaker $\mathrm{H} 3 \mathrm{~K} 4 \mathrm{me} 3$ and $\mathrm{H} 4 \mathrm{Ac}$ were detected in CLDN4-repressed cells (SNU484, MKN1 and SNU638). These results indicate that a loss of repressive histone methylations (H3K27me3, H4K20me3) and a gain of active histone modifications (H3K4me3, H4Ac) are associated with increased CLDN4 expression in gastric cancer cells. These results also suggest that increased claudin-4 expression in gastric cancer cells compared with normal gastric tissues is associated with epigenetic derepression by loss of repressive histone methylations.

Interestingly, the CLDN4 promoter was bivalently marked with both H3K4me3 and H3K27me3 in CLDN4-repressed SNU638 cells showing low DNA methylation consistent with normal gastric tissues, whereas $\mathrm{H} 3 \mathrm{~K} 4 \mathrm{me} 3$ alone was found in NCI-N87 cells (Figure 4d). On the other hand, there was a difference in histone modifications between CLDN4repressed cancer cells. The H3Ac level was remarkably higher in MKN1 cells than in the other two CLDN4-repressed cell lines. Unexpectedly, histone 3 lysine 9 methylations (H3K9me2, H3K9me3) known to be linked to DNA methylation were lacking in the CLDN4 promoter of DNA-hypermethylated cells, implying that crosstalks between DNA methylation and $\mathrm{H} 3 \mathrm{~K} 9 \mathrm{me} 2$ or $\mathrm{H} 3 \mathrm{~K} 9 \mathrm{me} 3$ are unlikely in the regulation of CLDN4 expression in gastric cancer cells. 
a

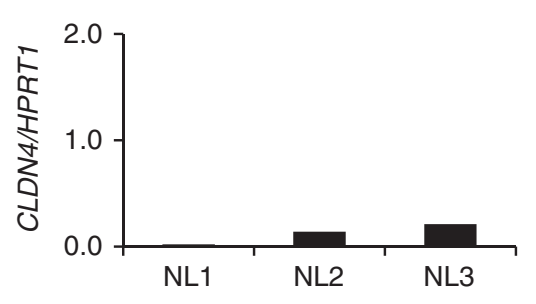

C

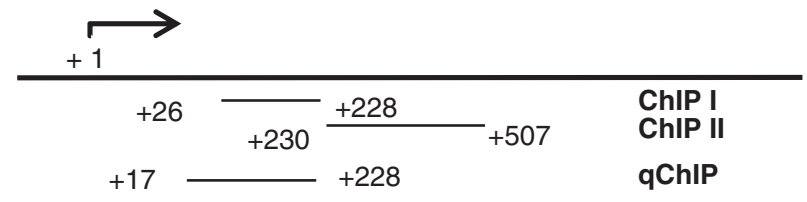

ChIP I (+26 +228)

ChIP II (+230 +507)

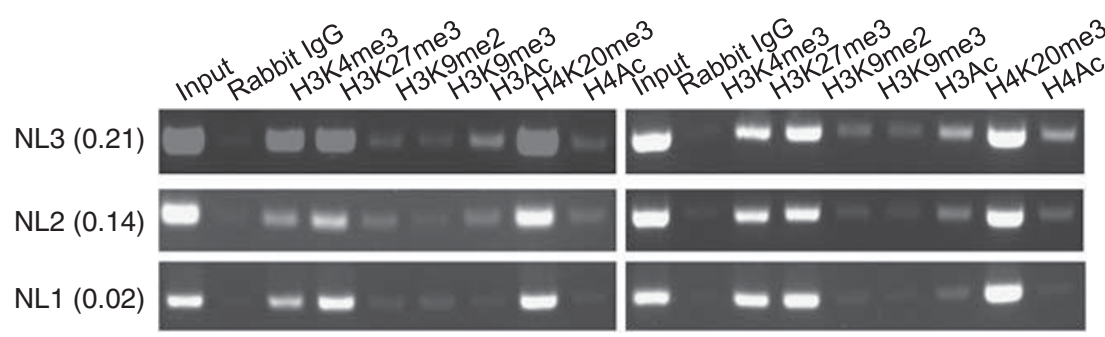

d

ChIP I (+26 +228)

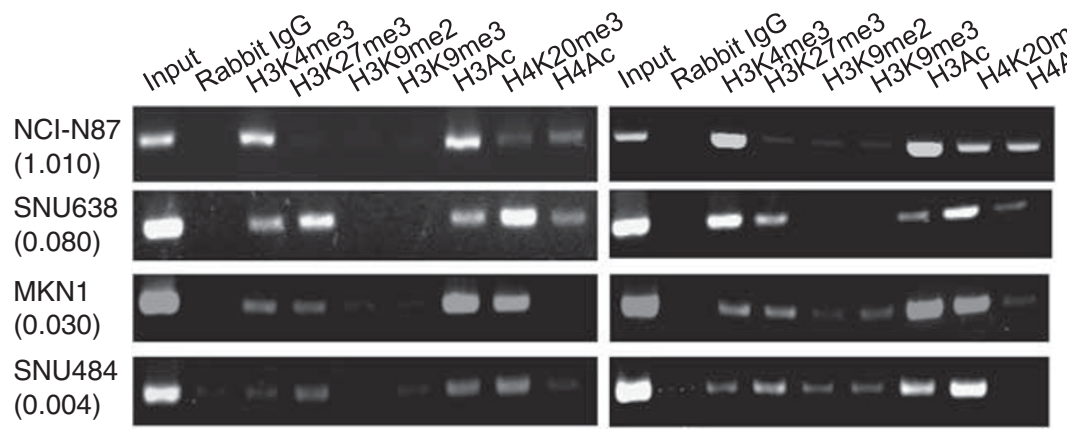

ChIP II (+230 +507)

$\begin{array}{lccc}\text { b } & +331 & +3 & +467 \\ \text { CpG site } & \mathbf{1} & \mathbf{1 0} & \mathbf{2 1}\end{array}$

NL3

NL2

NL1

$(\mathrm{n}=11)$
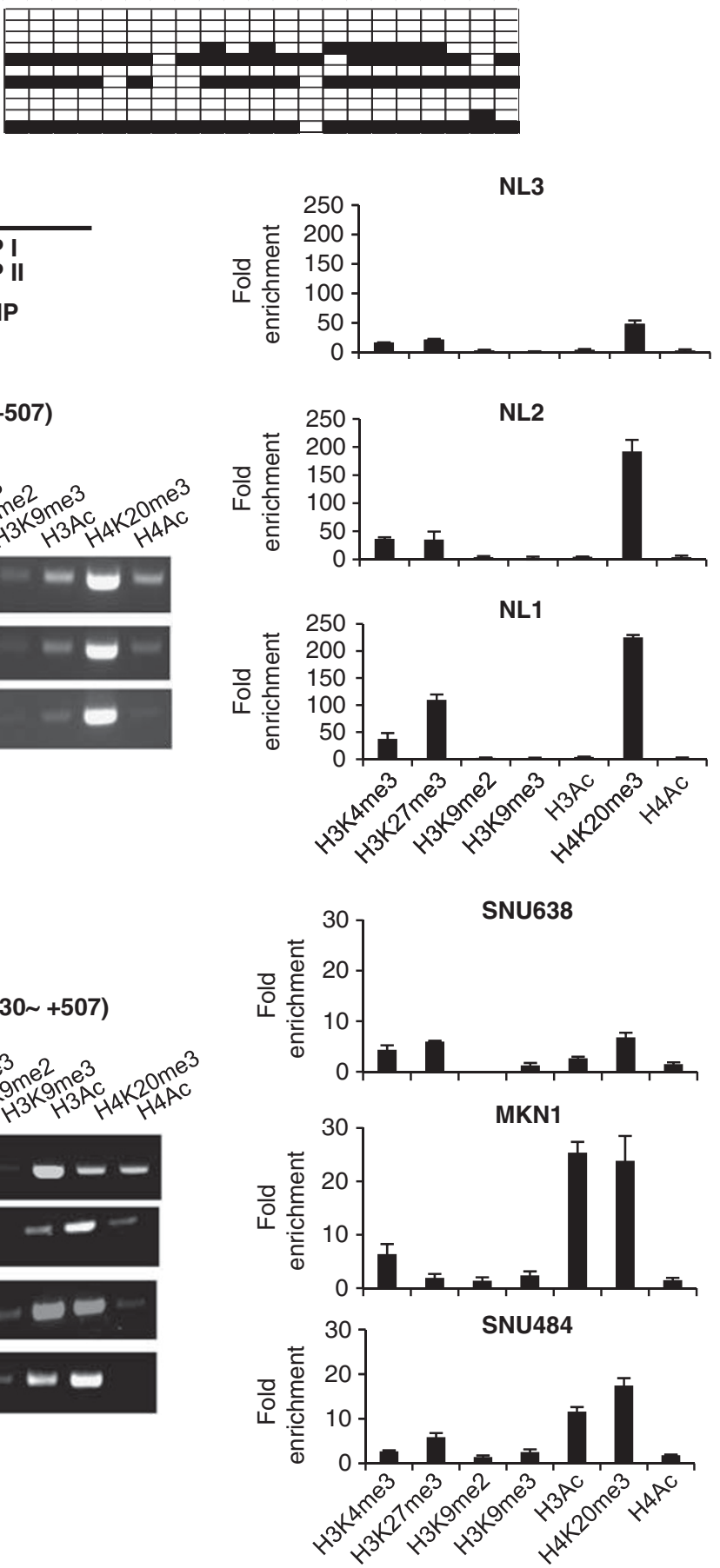


\section{CLDN4 Expression Is Differentially Regulated Depending on the Status of DNA Methylation and Histone Modifications in Gastric Cancer Cells}

Distinct patterns of histone modifications and DNA methylation levels at the promoter of CLDN4-repressed cells suggested the possibility of a differential response of CLDN4 expression to various epigenetic drug treatments. CLDN4repressed cancer cells were treated with epigenetic regulators 5 -aza-dC, TSA and DZNep alone and in various combinations, and the changes of CLDN4 expression in response to these treatments were assessed.

As expected, CLDN4 expression was significantly increased by 5-aza-dC alone (MKN1: 2.50-fold, SNU484: 2.95 -fold) in DNA-hypermethylated cells, whereas 5-aza-dC alone failed to induce CLDN4 expression in low DNA-methylated SNU638 cells (Figure 5a). However, CLDN4-repressed cells with DNA hypermethylation showed a differential response to TSA. CLDN4 expression was induced in response to TSA alone in SNU484 cells (2.32-fold), but was not induced in MKN1 cells. Moreover, the combination of 5-aza-dC and TSA (5-aza-dC/TSA) resulted in stronger synergic induction of CLDN4 expression in SNU484 (11.66- to 19.53-fold) than in MKN1 cells (3.33- to 5.50-fold). This might be due to the higher H3Ac level in MKN1 cells, suggesting that endogenous H3Ac level may affect the response to TSA. Notably, addition of DZNep to 5-aza-dC/TSA further enhanced CLDN4 expression compared with that from 5-aza-dC/TSA in DNAhypermethylated cells; however, DZNep alone did not reactivate CLDN4 expression.

We further assessed the changes in DNA methylation and each histone modification in cells treated with drugs compared with non-drug-treated cells to elucidate the mechanism through which CLDN4 expression is strongly derepressed in cells treated with combined treatments of DZNep, TSA or 5 -aza-dC. Increases in CLDN4 expression by 5 -aza-dC in MKN1 and SNU484 cells were accompanied by DNA demethylation (Figure 5b), showing that DNA demethylation is involved in induction of CLDN4 expression in the DNAhypermethylated promoter. In addition to DNA demethylation, simultaneous significant increases of active histone marks (H3K4me3, H3Ac and $\mathrm{H} 4 \mathrm{Ac}$ ) and decreases of repressive histone marks (H3K27me3, H4K20me3 and H3K9me3) were observed in DZNep/5-aza-dC/TSA-treated SNU484 cells compared with the level in no-treatment cells $(P<0.05$, Figure 5c, upper). 5-Aza-dC/TSA further significantly decreased $\mathrm{H} 4 \mathrm{~K} 20 \mathrm{me} 3$ and $\mathrm{H} 3 \mathrm{~K} 9 \mathrm{me} 3$ along with a concomitant increase of H3Ac and H4Ac $(P<0.05$, Figure $5 \mathrm{c}$, lower), indicating that the synergic derepression of CLDN4 resulting from addition of TSA correlates with a reduction in repressive $\mathrm{H} 4 \mathrm{~K} 20 \mathrm{me} 3$ and $\mathrm{H} 3 \mathrm{~K} 9 \mathrm{me} 3$ along with an increase in histone acetylation.

In contrast to CLDN4-repressed cells with DNA hypermethylation, CLDN4 expression was strongly reactivated by the combined treatment of DZNep and TSA (DZNep/TSA) in SNU638 cells (Figure 5a), indicating that histone modification rather than DNA methylation has a dominant role in the regulation of CLDN4 expression in cells with low DNA methylation. This is in line with a previous study, ${ }^{40}$ which showed that DZNep can reactivate genes not silenced by DNA methylation. However, the minimal induction of CLDN4 expression by DZNep alone, in contrast to the remarkable increase by DZNep/TSA treatment suggests that simultaneous changes in both histone methylations and histone acetylations, possibly through their crosstalks are required for strong reactivation of CLDN4 expression. A synergic increase in CLDN4 expression by DZNep/TSA compared with DZNep or TSA alone was also shown in MKN1 cells, suggesting the possibility of crosstalk between histone methylations and acetylation in this cell line as well. Furthermore, CLDN4 expression was not induced in SNU638 cells treated with EZH2 siRNA, despite a marked depletion of EZH2 expression along with a corresponding decrease in H3K27me3 (Figure 5d), supporting that the loss of the H3K27me3 mark alone is insufficient for re-expression of CLDN4 in this cell line. This also suggests that CLDN4 is not a direct target of EZH2 in contrast to RUNX3 or E-cadherin the expressions of which have been reported to be directly regulated by EZH2. ${ }^{41,42}$

As shown in Figure 5e, DZNep/TSA caused a significant increase in active $\mathrm{H} 3 \mathrm{~K} 4 \mathrm{me} 3, \mathrm{H} 3 \mathrm{Ac}$ and $\mathrm{H} 4 \mathrm{Ac}$ along with producing a significant reduction in H4K20me3 and H3K9me3 levels in SNU638 cells $(P<0.05)$. Unexpectedly, H3K27me3 was not decreased by DZNep/TSA, suggesting that loss of the EZH2-mediated H3K27me3 mark in SNU638 cells may not be responsible for CLDN4 derepression by DZNep/TSA.

Taken together, these data show that CLDN4 expression is differently regulated depending on the status of DNA

Figure 4 Histone modification status at the CLDN4 promoter in normal gastric tissues and gastric cancer cell lines. (a) CLDN4 expression in normal frozen gastric tissues. Transcript levels of three normal frozen tissues (NL1, 2, 3) were determined by qRT-PCR. (b) DNA methylation status at the CLDN4 promoter of normal gastric tissues. DNA methylation of $21 \mathrm{CpG}$ sites in the region from +192 to +147 was assessed by BSP. (c) Histone modification status at the CLDN4 promoter of normal gastric tissues and (d) of gastric cancer cell lines. ChIP analyses were performed in two regions (ChIP I to ChIP II) (right) to determine histone modifications at the CLDN4 promoter in three normal tissues and four cancer cell lines (1) CLDN4-highly expressing NCI-N87 cells having an DNA-unmethylated CLDN4 promoter, (2) CLDN4-repressed SNU638 cells with a low level of DNA methylation and (3) CLDN4-repressed cell lines with DNA hypermethylation (MKN1, SNU484). The transcript level of CLDN4 is indicated under the name of each cell line. The status of histone modifications was also quantitatively examined by qPCR (right). The data of qChIP are represented as fold enrichment values that are calculated by dividing the relative enrichment to the input amount by normal rabbit IgG value. 
methylation and histone modifications, and concomitant increases in active histone modifications and decreases in repressive histone methylations are required for strong derepression of CLDN4, along with DNA demethylation in CLDN4-hypermethylated gastric cancer cells.
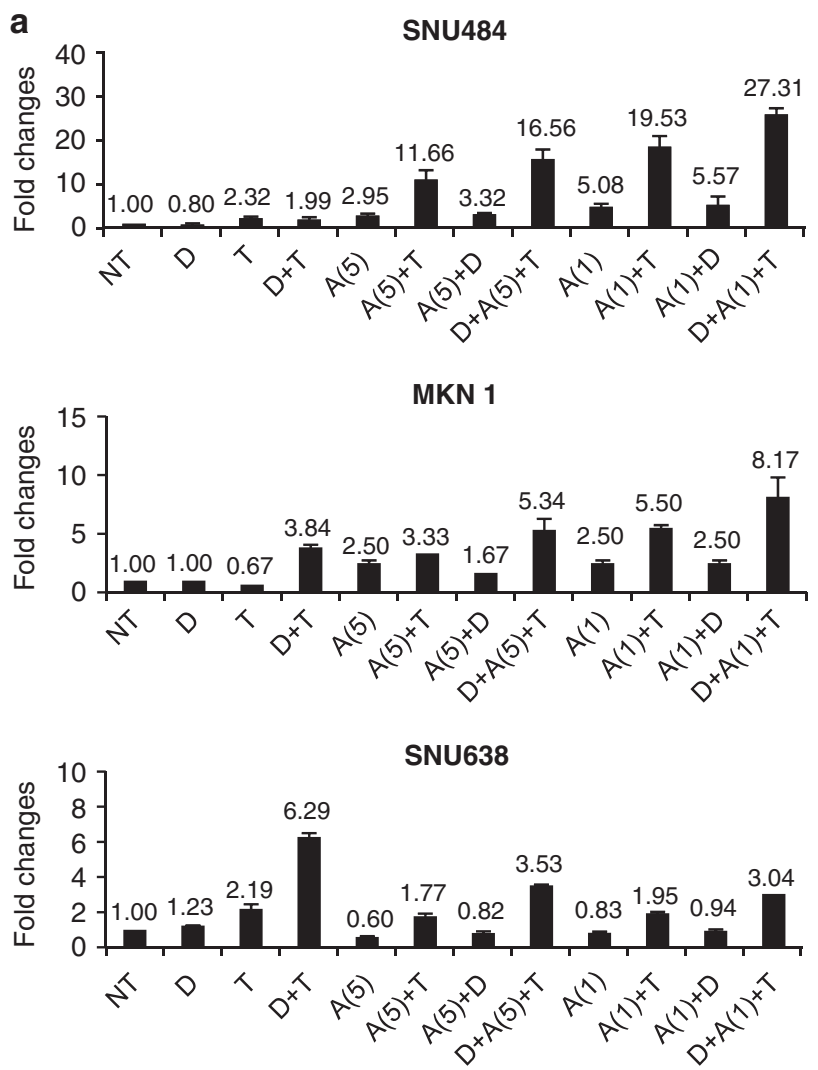

d

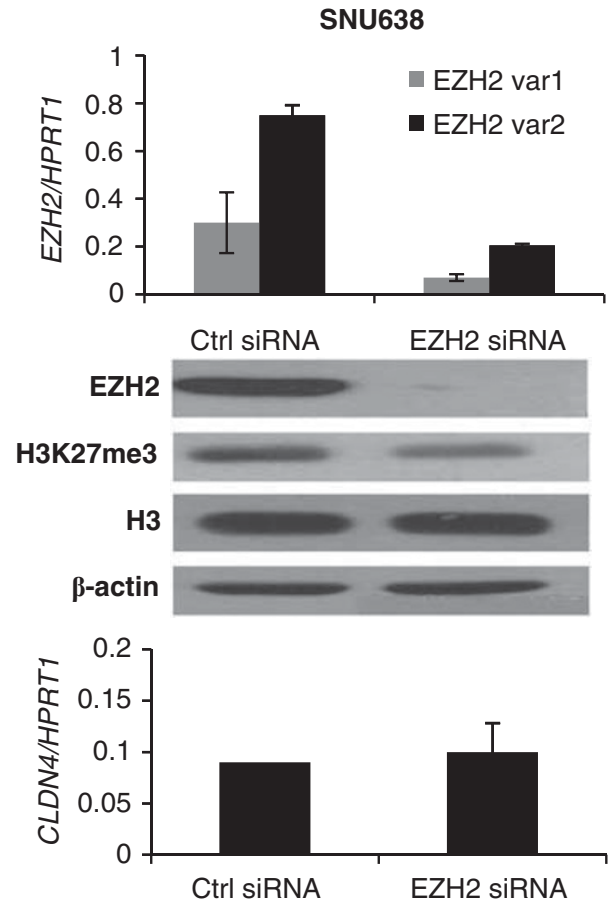

\section{DISCUSSION}

Despite the importance of claudin-4 as a promising therapeutic target for human cancer, the clinical implications of aberrant claudin-4 expression in various cancers and the molecular mechanisms leading to its dysregulation during

b

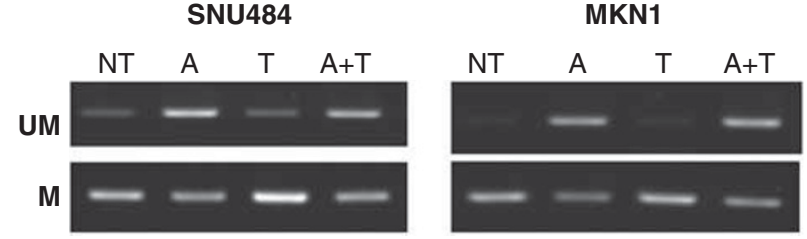

C
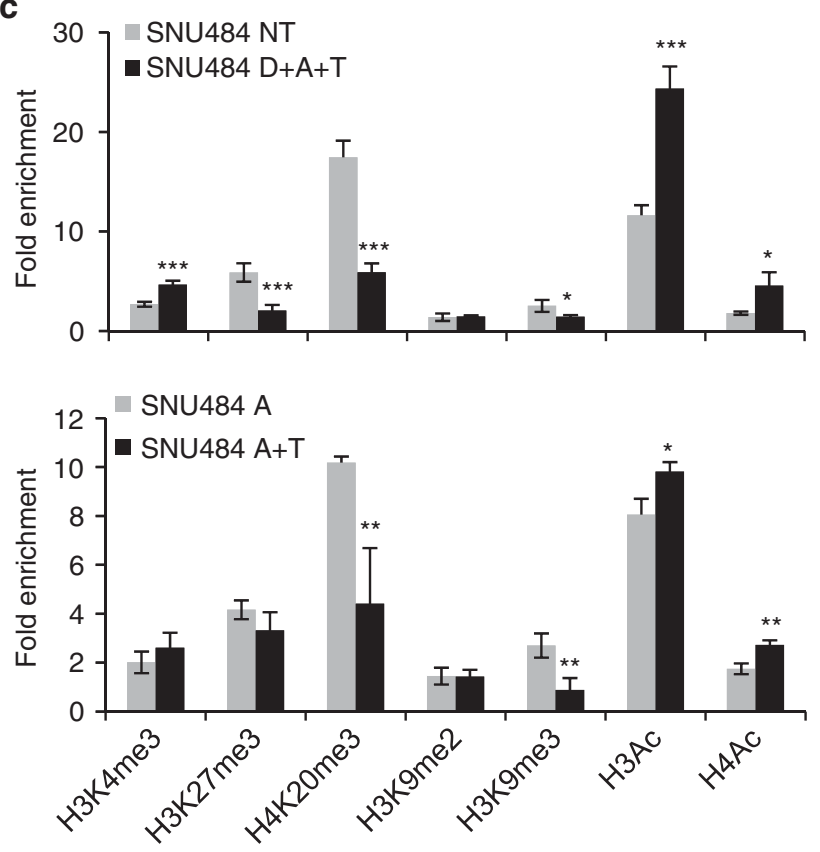

e

SNU638

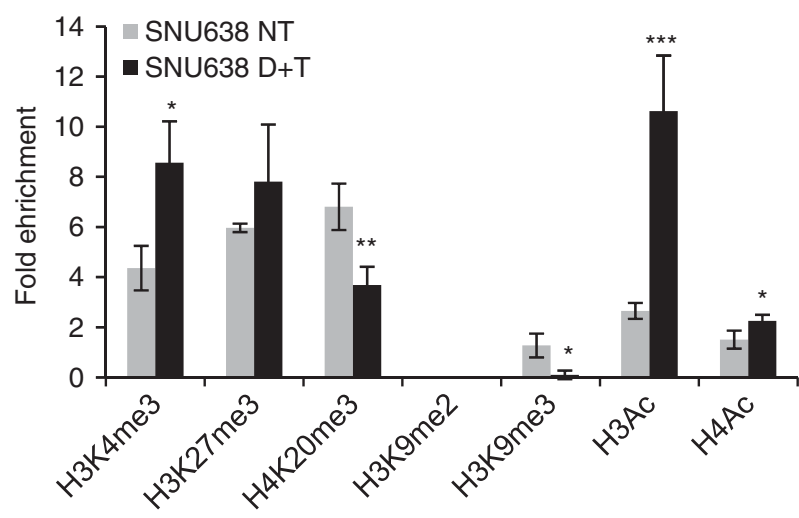


tumorigenesis have remained largely unknown This study elucidated an epigenetic mechanism leading to the dysregulation of CLDN4 expression in gastric cancer and the significance of claudin-4 overexpression in gastric cancer progression.

We show that increased membranous claudin- 4 expression is associated with better prognosis in contrast to no significant relationship between cytoplasmic claudin- 4 expression and prognosis of gastric cancer patients. A recent study by Ohtani $e t a l^{25}$ also reported a positive correlation between claudin-4 expression and prognosis; however, they assessed the relationship of total (membranous and cytoplasmic) claudin-4 expression with clinicopathological factors. We also observed that increased membranous claudin- 4 expression in gastric cancer cells inhibits cell migration and invasion, whereas it does not affect cell growth. These findings are consistent with a significant inverse correlation of membranous claudin-4 expression with tumor invasion and metastasis in our clinicopathological data and a previous report, which showed inhibition of cell migration and anchorageindependent growth related to claudin-4 overexpression in the gastric cancer cell line AGS. ${ }^{43}$ In accordance with previous findings in pancreatic cancer, ${ }^{22}$ the inhibition of cell migration and invasion by claudin- 4 expression observed in this study is considered to be associated with an increase in cell attachments between tumor cells because overexpressed claudin-4 was localized in the cell-cell contact region, whereas decreased invasion activity was not related to MMP activities. However, the effect of cellular localization of claudin-4 on migration or invasion in gastric cancer needs to be further investigated to clarify whether localization of claudin-4 affects its function.

Our data also show that claudin-4 overexpression increases the barrier function of TJs in gastric cancer cells, results that are consistent with previous studies in colon cancer ${ }^{44}$ and MDCK cells, ${ }^{45}$ thus, suggesting that overexpression of claudin-4 might suppress gastric cancer progression by enhancing the barrier function of TJs as well as by inhibiting cell migration and invasion.

It is notable that claudin-4 expression has opposite roles in cancer progression in a tissue-specific manner. In contrast to no relationship between MMP activity and cell invasion by claudin- 4 expression in this study, claudin- 4 expression was reported to enhance cell invasion by an increase of MMP-2 activity in ovarian cells. ${ }^{8}$ Furthermore, although this study showed that claudin-4 expression increases the barrier function of TJs in gastric cancer cells, claudin-4 expression has not been associated with $\mathrm{TJ}$ integrity in ovarian cancer cells. ${ }^{13}$ These results suggest that claudin- 4 might promote tumorigenesis or may suppress tumorigenesis by different molecular mechanisms in association with tissue-specific circumstances. Similarly, claudin-1 has shown a tumorsuppressive activity in gastric cancer, ${ }^{46}$ in contrast to its cancer-promoting role in colon cancer. ${ }^{10}$

Epigenetic silencing of multiple genes by frequent $\mathrm{CpG}$ island hypermethylation in early gastric tumorigenesis and gastric carcinoma has been reported; ${ }^{29,30}$ however, the upregulation of gene expression by such an epigenetic mechanism is not well known. Here, we demonstrate that upregulation of claudin- 4 expression during multistep gastric tumor progression is associated with promoter DNA hypomethylation. Moreover, DNA hypomethylation was significantly correlated with high claudin-4 expression in gastric carcinoma, and decreased claudin-4 expression in advanced stages of gastric cancer was significantly associated with increased promoter DNA methylation, as has been similarly reported in ovarian ${ }^{47}$ and bladder carcinoma, ${ }^{21}$ suggesting that DNA methylation is an important mechanism regulating CLDN4 expression in gastric carcinogenesis (Supplementary Figure S3a).

Furthermore, these results demonstrate that claudin-4 expression is repressed in normal gastric tissues in association with bivalent histone modifications, and loss of repressive histone methylations is also associated with claudin- 4 overexpression in gastric cancer cells consistent with our previous results in ovarian cancer cells. ${ }^{33}$ However, this study shows that CLDN4 expression may be differentially regulated, depending on the status of both DNA methylation and histone modifications. That is, CLDN4 repression with DNA hypermethylation could be strongly reversed by simultaneous DNA demethylation and histone acetylation, whereas concurrent loss of repressive histone methylation and gain of active histone modifications, are required for strong induction of CLDN4 expression in gastric cancer cells with low DNA methylation (Supplementary Figure S3b). This suggests that epigenetic derepression by simultaneous changes in

Figure 5 Changes in CLDN4 expression and corresponding epigenetic modifications in response to DZNep, 5-aza-dC, TSA and their combinations in gastric cancer cell lines. (a) Changes in CLDN4 expression compared with no treatment (NT) after drug treatments. SNU484, MKN1 and SNU638 cells were treated with $5 \mu \mathrm{M}$ DZNep (D) for $72 \mathrm{~h}, 200-300 \mathrm{nM}$ TSA for $24 \mathrm{~h}$ (T) and $1 \mu \mathrm{M}$ 5-aza-dC (A) for $96 \mathrm{~h}$ or with $5 \mu \mathrm{M}$ for $72 \mathrm{~h}$ either alone or in indicated combinations. For co-treatment of cells with DZNep or $5 \mu \mathrm{M} \mathrm{5}$-aza-dC and TSA, DZNep or $5 \mu \mathrm{M} 5$-aza-dC were added for $48 \mathrm{~h}$ followed by TSA for an additional $24 \mathrm{~h}$ for gene expression analysis. For co-treatment of cells with $1 \mu \mathrm{M}$ 5-aza-dC and TSA, $1 \mu \mathrm{M}$-aza-dC was added for $72 \mathrm{~h}$ followed by TSA for an additional $24 \mathrm{~h}$ for gene expression analysis. CLDN4 expression was analyzed by qRT-PCR. $Y$ axes indicate the folds of induction relative to untreated cells. (b) Changes in DNA methylation status after 5-aza-dC or 5-aza-dC and TSA combination treatment. UM and M were determined by MSP analyses. (c) ChIP analysis showing changes in histone modifications at the CLDN4 promoter after epigenetic drug treatments in SNU484 cells. (d) Effect of EZH2 knockdown on CLDN4 expression in SNU638 cells. Knockdown of EZH2 transcripts (EZH2 var1 and EZH2 var2) (top) and the decrease in protein levels of EZH2 and H3K27me3 (middle) were assessed. CLDN4 mRNA levels after EZH2 siRNA treatments were compared with the levels after control siRNA (ctrl siRNA) treatment (bottom). (e) Changes in histone modifications after combined treatment of DZNep and TSA compared with no treatment in SNU638 cells. Mean \pm s.d., ${ }^{*} P \leq 0.05$, ${ }^{* *} P \leq 0.01,{ }^{* * *} P \leq 0.001$ (Student's $t$-test). 
histone modifications and through DNA demethylation might be one possible mechanism underlying claudin-4 overexpression in gastric cancer. On the basis of the epigenetic derepression mechanism in the upregulation of claudin-4 in gastric cancer cells, it is possible that epigenetic treatments can be used in combination with other therapies such as therapeutic antibody targeting claudin- 4 for the effective treatment of gastric cancer by enhancing sensitivity to those therapies.

In addition to such an epigenetic mechanism, several transcription factors have been known to be involved in the regulation of claudin- 4 gene expression. Cdx2, a transcription factor inducing intestinal metaplasia in the gastric epithelium, ${ }^{31}$ has positively regulated the expression of claudin4 during gastric carcinoma intestinal differentiation..$^{39,48}$ Snail and twist that have a central role in the epitheliummesenchymal transition (EMT), have been shown to repress claudin-4 expression in mouse epithelial cells, ${ }^{49}$ colorectal cancer cells ${ }^{50}$ and breast cancer cells. ${ }^{51}$ In addition, tumorigenesis-associated TGF- $\beta$ and Ras signaling pathways were reported to affect $C L D N 4$ expression in pancreatic cancer cells. ${ }^{22}$ However, another mechanism seems to be involved in the regulation of CLDN4 expression in gastric cancer cells because they were not well correlated with claudin-4 expression in gastric cancer cell lines (Supplementary Figure S4). As transcription factors are known to cooperate with epigenetic mechanism to regulate gene expression, further studies on interactions between CLDN4-relevant epigenetic mechanism and transcription factors will allow for better understanding on the mechanism regulating CLDN4 expression in gastric cancer.

In particular, the previously reported claudin-4 downregulation during snail-induced EMT, which is a key step for cancer invasion and metastasis, ${ }^{49}$ suggests that claudin-4 may have a role in cancer progression through EMT. Actually, EMT characteristics, including the loss of E-cadherin expression and the appearance of expression of the mesenchymal marker vimentin were observed in gastric cancer cells with low claudin-4 expression (Supplementary Figure S5). On the other hand, claudin-1 expression in colon cancer was shown to induce changes in EMT markers. ${ }^{10}$ However, it is unclear whether claudin- 4 actually contributes to EMT in gastric cancer; thus, further studies are required to elucidate the role of claudin-4 in EMT.

In summary, this study demonstrates that membranous claudin-4 expression is an independent positive prognostic factor in gastric carcinoma and that it inhibits the migration and invasion of gastric cancer cells. It was elucidated that claudin-4 overexpression in gastric cancer cells is associated with epigenetic derepression through simultaneous changes in histone modifications and/or DNA demethylation. Our findings suggest that claudin- 4 is a promising target for the treatment of gastric cancer, and increase of claudin-4 by epigenetic derepression may contribute to the suppression of gastric cancer progression.
Supplementary Information accompanies the paper on the Laboratory Investigation website (http://www.laboratoryinvestigation.org)

\section{ACKNOWLEDGEMENTS}

We thank Dr Victor E Marquez for DZNep and we are grateful to Si Eun Kim for her technical assistance. This study was supported by grants from the Korea Healthcare Technology R\&D project of the Ministry of Health, Welfare \& Family Affairs (A084366), the National Research Foundation of Korea (NRF) by the Korea government (MEST) (No.20090083533) and the R\&D program of MKE/KEIT (10035329, Development of Antibody drug for new target protein related with ovarian cancer).

\section{DISCLOSURE/CONFLICT OF INTEREST}

The authors declare no conflict of interest.

1. Morin PJ. Claudin proteins in human cancer: promising new targets for diagnosis and therapy. Cancer Res 2005;65:9603-9606.

2. Shin K, Fogg VC, Margolis B. Tight junctions and cell polarity. Annu Rev Cell Dev Biol 2006;22:207-235.

3. Lal-Nag M, Morin PJ. The claudins. Genome Biol 2009;10:235.

4. Hewitt KJ, Agarwal R, Morin PJ. The claudin gene family: expression in normal and neoplastic tissues. BMC Cancer 2006;6:186.

5. Kominsky SL. Claudins: emerging targets for cancer therapy. Expert Rev Mol Med 2006;8:1-11.

6. Darido C, Buchert M, Pannequin J, et al. Defective claudin-7 regulation by Tcf-4 and Sox-9 disrupts the polarity and increases the tumorigenicity of colorectal cancer cells. Cancer Res 2008;68: 4258-4268.

7. Oku N, Sasabe E, Ueta $E$, et al. Tight junction protein claudin-1 enhances the invasive activity of oral squamous cell carcinoma cells by promoting cleavage of laminin-5 gamma2 chain via matrix metalloproteinase (MMP)-2 and membrane-type MMP-1. Cancer Res 2006;66:5251-5257.

8. Agarwal R, D'Souza T, Morin PJ. Claudin-3 and claudin-4 expression in ovarian epithelial cells enhances invasion and is associated with increased matrix metalloproteinase-2 activity. Cancer Res 2005;65:7378-7385.

9. Lioni $M$, Brafford $P$, Andl C, et al. Dysregulation of claudin-7 leads to loss of E-cadherin expression and the increased invasion of esophageal squamous cell carcinoma cells. Am J Pathol 2007;170:709-721.

10. Dhawan $P$, Singh $A B$, Deane NG, et al. Claudin-1 regulates cellular transformation and metastatic behavior in colon cancer. J Clin Invest 2005;115:1765-1776.

11. Michl P, Buchholz $M$, Rolke $M$, et al. Claudin-4: a new target for pancreatic cancer treatment using Clostridium perfringens enterotoxin. Gastroenterology 2001;121:678-684.

12. Long $\mathrm{H}, \mathrm{Crean} C D$, Lee $W H$, et al. Expression of Clostridium perfringens enterotoxin receptors claudin-3 and claudin-4 in prostate cancer epithelium. Cancer Res 2001;61:7878-7881.

13. Rangel LB, Agarwal R, D'Souza T, et al. Tight junction proteins claudin-3 and claudin-4 are frequently overexpressed in ovarian cancer but not in ovarian cystadenomas. Clin Cancer Res 2003;9:2567-2575.

14. Kominsky SL, Vali M, Korz D, et al. Clostridium perfringens enterotoxin elicits rapid and specific cytolysis of breast carcinoma cells mediated through tight junction proteins claudin 3 and 4. Am J Pathol 2004;164:1627-1633.

15. Choi YL, Kim J, Kwon MJ, et al. Expression profile of tight junction protein claudin 3 and claudin 4 in ovarian serous adenocarcinoma with prognostic correlation. Histol Histopathol 2007;22:1185-1195.

16. Cunningham SC, Kamangar F, Kim MP, et al. Claudin-4, mitogenactivated protein kinase kinase 4 , and stratifin are markers of gastric adenocarcinoma precursor lesions. Cancer Epidemiol Biomarkers Prev 2006;15:281-287.

17. Soini $\mathrm{Y}$, Tommola $\mathrm{S}$, Helin $\mathrm{H}$, et al. Claudins $1,3,4$ and 5 in gastric carcinoma, loss of claudin expression associates with the diffuse subtype. Virchows Arch 2006:448:52-58.

18. Kuo WL, Lee LY, Wu CM, et al. Differential expression of claudin-4 between intestinal and diffuse-type gastric cancer. Oncol Rep 2006; 16:729-734. 
19. Lee SK, Moon J, Park SW, et al. Loss of the tight junction protein claudin 4 correlates with histological growth-pattern and differentiation in advanced gastric adenocarcinoma. Oncol Rep 2005; 13:193-199.

20. Lanigan F, McKiernan E, Brennan DJ, et al. Increased claudin-4 expression is associated with poor prognosis and high tumour grade in breast cancer. Int J Cancer 2009;124:2088-2097.

21. Boireau S, Buchert M, Samuel MS, et al. DNA-methylation-dependent alterations of claudin-4 expression in human bladder carcinoma. Carcinogenesis 2007;28:246-258.

22. Michl P, Barth C, Buchholz M, et al. Claudin-4 expression decreases invasiveness and metastatic potential of pancreatic cancer. Cancer Res 2003;63:6265-6271.

23. Li J, Chigurupati $\mathrm{S}$, Agarwal $\mathrm{R}$, et al. Possible angiogenic roles for claudin-4 in ovarian cancer. Cancer Biol Ther 2009;8 1806-1814.

24. Resnick MB, Gavilanez M, Newton E, et al. Claudin expression in gastric adenocarcinomas: a tissue microarray study with prognostic correlation. Hum Pathol 2005;36:886-892.

25. Ohtani S, Terashima M, Satoh J, et al. Expression of tight-junctionassociated proteins in human gastric cancer: downregulation of claudin-4 correlates with tumor aggressiveness and survival. Gastric Cancer 2009;12:43-51.

26. Baylin SB, Ohm JE. Epigenetic gene silencing in cancer - a mechanism for early oncogenic pathway addiction? Nat Rev Cancer 2006;6: 107-116.

27. Esteller M. Epigenetics in cancer. N Engl J Med 2008;358:1148-1159.

28. Esteller M. Cancer epigenomics: DNA methylomes and histonemodification maps. Nat Rev Genet 2007;8:286-298.

29. Kang GH, Shim $\mathrm{YH}$, Jung $\mathrm{HY}$, et al. CpG island methylation in premalignant stages of gastric carcinoma. Cancer Res 2001;61: 2847-2851.

30. Choi IS, Wu TT. Epigenetic alterations in gastric carcinogenesis. Cell Res 2005;15:247-254.

31. Yuasa Y. Control of gut differentiation and intestinal-type gastric carcinogenesis. Nat Rev Cancer 2003;3:592-600.

32. Honda $\mathrm{H}$, Pazin $\mathrm{MJ}, \mathrm{Ji} \mathrm{H}$, et al. Crucial roles of $\mathrm{Sp} 1$ and epigenetic modifications in the regulation of the CLDN4 promoter in ovarian cancer cells. J Biol Chem 2006;281:21433-21444.

33. Kwon MJ, Kim SS, Choi YL, et al. Derepression of CLDN3 and CLDN4 during ovarian tumorigenesis is associated with loss of repressive histone modifications. Carcinogenesis 2010;31:974-983.

34. Wang $\mathrm{LH}, \mathrm{Kim} \mathrm{SH}$, Lee $\mathrm{JH}$, et al. Inactivation of SMAD4 tumor suppressor gene during gastric carcinoma progression. Clin Cancer Res 2007;13:102-110.

35. Sinicrope FA, Ruan SB, Cleary KR, et al. bcl-2 and p53 oncoprotein expression during colorectal tumorigenesis. Cancer Res 1995;55:237-241.

36. Kwon MJ, Oh E, Lee $\mathrm{S}$, et al. Identification of novel reference genes using multiplatform expression data and their validation for quantitative gene expression analysis. PLoS One 2009;4:e6162.
37. Johnson $\mathrm{AH}$, Frierson $\mathrm{HF}$, Zaika $\mathrm{A}$, et al. Expression of tight-junction protein claudin-7 is an early event in gastric tumorigenesis. Am J Pathol 2005;167:577-584.

38. Sanada $Y$, Oue N, Mitani $Y$, et al. Down-regulation of the claudin-18 gene, identified through serial analysis of gene expression data analysis, in gastric cancer with an intestinal phenotype. J Pathol 2006;208:633-642.

39. Matsuda $Y$, Semba $S$, Ueda J, et al. Gastric and intestinal claudin expression at the invasive front of gastric carcinoma. Cancer Sci 2007;98:1014-1019.

40. Miranda TB, Cortez CC, Yoo CB, et al. DZNep is a global histone methylation inhibitor that reactivates developmental genes not silenced by DNA methylation. Mol Cancer Ther 2009;8:1579-1588.

41. Fujii S, Ito $K$, Ito $Y$, et al. Enhancer of zeste homologue 2 (EZH2) downregulates RUNX3 by increasing histone $\mathrm{H} 3$ methylation. J Biol Chem 2008;283:17324-17332.

42. Fujii S, Ochiai A. Enhancer of zeste homolog 2 downregulates E-cadherin by mediating histone $\mathrm{H} 3$ methylation in gastric cancer cells. Cancer Sci 2008;99:738-746.

43. Mima S, Tsutsumi S, Ushijima $\mathrm{H}$, et al. Induction of claudin-4 by nonsteroidal anti-inflammatory drugs and its contribution to their chemopreventive effect. Cancer Res 2005;65:1868-1876.

44. Takehara M, Nishimura T, Mima S, et al. Effect of claudin expression on paracellular permeability, migration and invasion of colonic cancer cells. Biol Pharm Bull 2009;32:825-831.

45. Van Itallie C, Rahner C, Anderson JM. Regulated expression of claudin-4 decreases paracellular conductance through a selective decrease in sodium permeability. J Clin Invest 2001;107:1319-1327.

46. Chang TL, Ito K, Ko TK, et al. Claudin-1 has tumor suppressive activity and is a direct target of RUNX3 in gastric epithelial cells. Gastroenterology 2010;138:255-265.

47. Litkouhi B, Kwong J, Lo CM, et al. Claudin-4 overexpression in epithelial ovarian cancer is associated with hypomethylation and is a potential target for modulation of tight junction barrier function using a C-terminal fragment of Clostridium perfringens enterotoxin. Neoplasia 2007;9:304-314

48. Satake $\mathrm{S}$, Semba $\mathrm{S}$, Matsuda $\mathrm{Y}$, et al. $\mathrm{Cdx} 2$ transcription factor regulates claudin-3 and claudin-4 expression during intestinal differentiation of gastric carcinoma. Pathol Int 2008;58:156-163.

49. Ikenouchi J, Matsuda M, Furuse $M$, et al. Regulation of tight junctions during the epithelium-mesenchyme transition: direct repression of the gene expression of claudins/occludin by Snail. J Cell Sci 2003;116 (Pt 10):1959-1967.

50. De Craene B, Gilbert B, Stove $C$, et al. The transcription factor snail induces tumor cell invasion through modulation of the epithelial cell differentiation program. Cancer Res 2005;65: 6237-6244.

51. Mironchik Y, Winnard Jr PT, Vesuna F, et al. Twist overexpression induces in vivo angiogenesis and correlates with chromosomal instability in breast cancer. Cancer Res 2005;65:10801-10809. 